\title{
HUBBLE TARANTULA TREASURY PROJECT. III. PHOTOMETRIC CATALOG AND RESULTING CONSTRAINTS ON THE PROGRESSION OF STAR FORMATION IN THE 30 DORADUS REGION*
}

\author{
E. Sabbi ${ }^{1}$, D. J. Lennon $^{2}$, J. Anderson ${ }^{1}$, M. Cignoni ${ }^{1}$, R. P. van der Marel ${ }^{1}$, D. Zaritsky ${ }^{3}$, G. De Marchi ${ }^{4}$, N. Panagia ${ }^{1,5,6}$ \\ D. A. Gouliermis ${ }^{7,8}$, E. K. Grebel ${ }^{9}$, J. S. Gallagher III ${ }^{10}$, L. J. Smith ${ }^{11}$, H. Sana ${ }^{1}$, A. Aloisi ${ }^{1}$, M. Tosi ${ }^{12}$, C. J. Evans ${ }^{13}$, \\ H. Arab ${ }^{1}$, M. Boyer ${ }^{14,15}$, S. E. De Mink ${ }^{16}$, K. Gordon ${ }^{1}$, A. M. KoeKemoer ${ }^{1}$, S. S. LARSEN ${ }^{17}$, J. E. RyON ${ }^{10}$, AND P. ZeidleR ${ }^{9}$ \\ ${ }^{1}$ Space Telescope Science Institute, 3700 San Martin Drive, Baltimore, MD, 21218, USA; sabbi@ \\ ${ }^{2}$ ESA-European Space Astronomy Center, Apdo. de Correo 78, E-28691 Associate Villanueva de la Cañada, Madrid, Spain \\ ${ }^{3}$ Steward Observatory, University of Arizona, 933 North Cherry Avenue, Tucson, AZ 85721, USA \\ ${ }^{4}$ Space Science Department, European Space Agency, Keplerlaan 1, 2200 AG Noordwijk, The Netherlands \\ ${ }^{5}$ Istituto Nazionale di Astrofisica, Osservatorio Astrofisico di Catania, Via Santa Sofia 78, I-95123 Catania, Italy \\ ${ }^{6}$ Supernova Limited, OYV 131, Northsound Road, Virgin Gorda, British Virgin Islands \\ ${ }^{7}$ Zentrum für Astronomie der Universität Heidelberg, Institut für Theoretische Astrophysik, Albert-Ueberle-Str. 2, D-69120 Heidelberg, Germany \\ ${ }^{8}$ Max-Planck-Institut für Astronomie, Königstuhl 17, D-69117 Heidelberg, Germany \\ ${ }^{9}$ Astronomisches Rechen-Institut, Zentrum für Astronomie der Universität Heidelberg, Mönchhofstr. 12-14, D-69120 Heidelberg, Germany \\ ${ }^{10}$ Department of Astronomy, University of Wisconsin, 475 North Charter Street, Madison, WI 53706, USA \\ ${ }^{11}$ ESA/STScI, 3700 San Martin Drive, Baltimore, MD, 21218, USA \\ ${ }^{12}$ Istituto Nazionale di Astrofisica, Osservatorio Astronomico di Bologna, Via Ranzani 1, I-40127 Bologna, Italy \\ ${ }_{13}^{13}$ UK Astronomy Technology Center, Royal Observatory Edinburgh, Blackford Hill, Edinburgh, EH9 3HJ, UK \\ ${ }^{14}$ Observational Cosmology Lab, Code 665, NASA, Goddard Space Flight Center, Greenbelt, MD, 20771, USA \\ ${ }^{15}$ Oak Ridge Associate Universities (ORAU), Oak Ridge, TN 37831, USA
Astronomical Institute “Anton Pannekoek,"University of Amsterdam, P.O. Box 94249, NL-1090 GE Amsterdam, The Netherlands \\ ${ }^{17}$ Department of Astrophysics/IMAPP, Radboud University Nijmegen, P.O. Box 9010, 6500 GL Nijmegen, The Netherlands \\ Received 2015 June 8; accepted 2015 November 17; published 2016 January 22
}

\begin{abstract}
We present and describe the astro-photometric catalog of more than 800,000 sources found in the Hubble Tarantula Treasury Project (HTTP). HTTP is a Hubble Space Telescope Treasury program designed to image the entire 30 Doradus region down to the sub-solar $\left(\sim 0.5 M_{\odot}\right)$ mass regime using the Wide Field Camera 3 and the Advanced Camera for Surveys. We observed 30 Doradus in the near-ultraviolet (F275W, F336W), optical (F555W, F658N, F775W), and near-infrared (F110W, F160W) wavelengths. The stellar photometry was measured using pointspread function fitting across all bands simultaneously. The relative astrometric accuracy of the catalog is 0.4 mas. The astro-photometric catalog, results from artificial star experiments, and the mosaics for all the filters are available for download. Color-magnitude diagrams are presented showing the spatial distributions and ages of stars within 30 Dor as well as in the surrounding fields. HTTP provides the first rich and statistically significant sample of intermediate- and low-mass pre-main sequence candidates and allows us to trace how star formation has been developing through the region. The depth and high spatial resolution of our analysis highlight the dual role of stellar feedback in quenching and triggering star formation on the giant $\mathrm{H}$ in region scale. Our results are consistent with stellar sub-clustering in a partially filled gaseous nebula that is offset toward our side of the Large Magellanic Cloud.
\end{abstract}

Key words: catalogs - galaxies: star clusters: individual (30 Doradus) - Magellanic Clouds - stars: formation stars: imaging - stars: pre-main sequence

\section{INTRODUCTION}

30 Doradus (also known as the Tarantula Nebula) in the Large Magellanic Cloud (LMC) is the most powerful source of $\mathrm{H} \alpha$ emission in the Local Group $(f(\mathrm{H} \alpha) \sim 1.3 \times$ $10^{-8} \mathrm{erg} \mathrm{cm}^{-2} \mathrm{~s}^{-1}$, Kennicutt \& Hodge 1986). Covering an area of $\sim 40,000 \mathrm{pc}^{2}, 30$ Doradus is the closest extragalactic giant $\mathrm{H}$ II region and is comparable in size to the unresolved luminous $\mathrm{H}_{\mathrm{II}}$ complexes observed in distant galaxies (Oey et al. 2003; Hunt \& Hirashita 2009). In terms of size $\left(\sim 200 \mathrm{pc}\right.$ in diameter) and stellar density (between $\rho_{0} \geqslant$ $1.5 \times 10^{4}-10^{7} M_{\odot} \mathrm{pc}^{-3}$, Selman \& Melnick 2013), the Nebula is often equated to regions of extreme star formation such as the starburst knots observed in interacting galaxies in the local

\footnotetext{
* Based on observations with the NASA/ESA Hubble Space Telescope, obtained at the Space Telescope Science Institute, which is operated by AURA Inc., under NASA contract NAS 5-26555.
}

universe and young galaxies at high redshift $(z>5$, Meurer et al. 1997; Shapley et al. 2003; Heckman et al. 2004).

Radcliffe 136 (R136), the core of 30 Doradus's ionizing cluster NGC 2070, contains the most massive stars $\left(\sim 300 M_{\odot}\right)$ known so far (Crowther et al. 2010; Bestenlehner et al. 2011) and is considered a testbed for understanding the early evolution of massive stars. Because of its mass $\left(\sim 10^{5} M_{\odot}\right.$, Selman et al. 1999; Bosch et al. 2001; Andersen et al. 2009; Cignoni et al. 2015), NGC 2070 has often been compared to young globular clusters.

By virtue of its location in the LMC ( $\sim 50 \mathrm{kpc}$; Panagia et al. 1991; Pietrzyński et al. 2013), the low inclination angle $\left(\sim 30^{\circ}\right.$; Nikolaev et al. 2004), and the low foreground reddening, 30 Doradus is an ideal target to study the process of massive star formation in detail. It is thus not surprising that the entire region has been surveyed by all the Great Observatories (Spitzer Space Telescope-Meixner et al. 2006; Herschel Space Observatory-Meixner et al. 2010; Chandra $X$-ray Observatory-Townsley et al. 2006 and the ongoing 


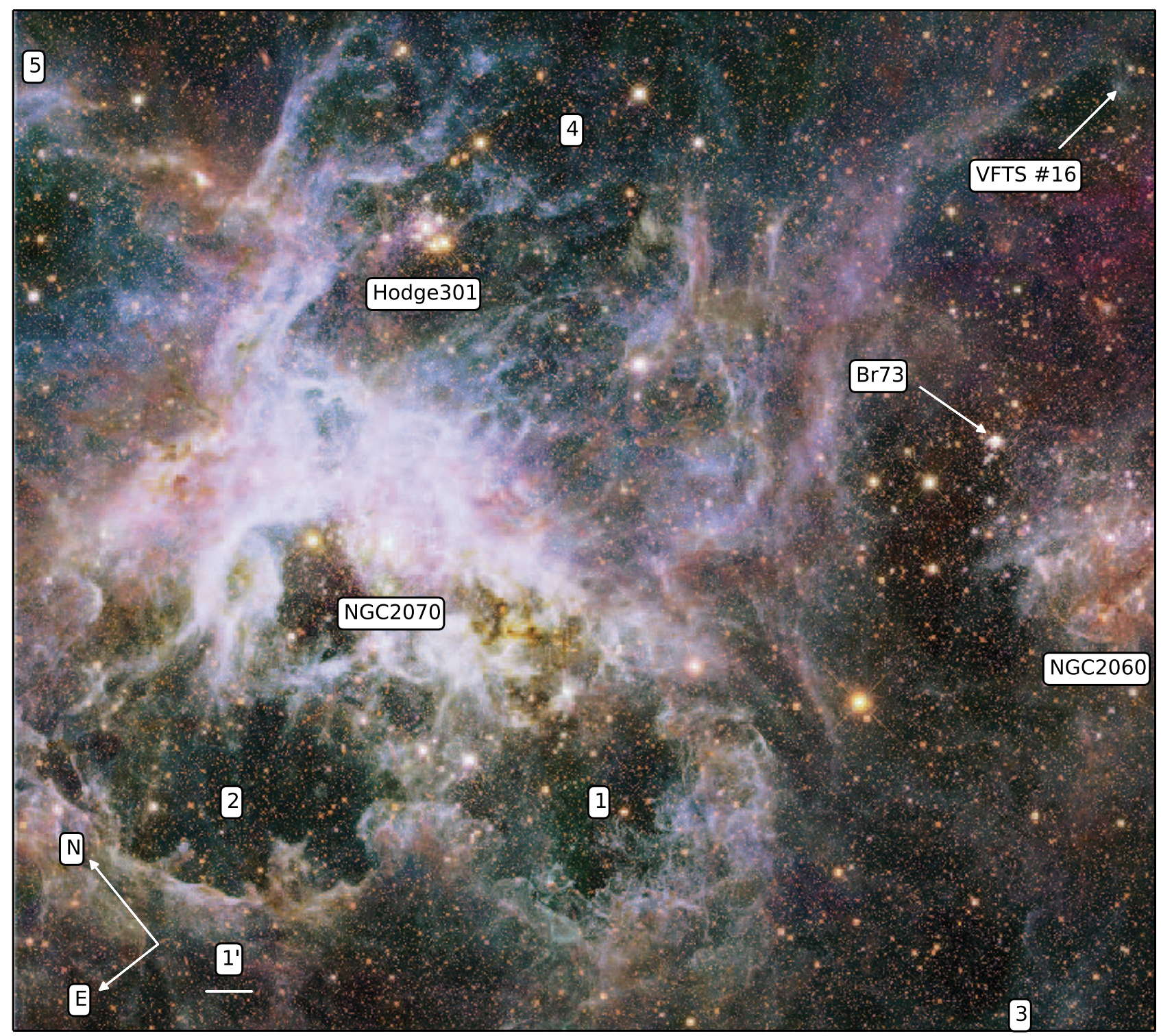

Figure 1. Color composite image of 30 Doradus in the light of F110W (in blue) and F160W (in red). The position of the four larger clusters and associations is highlighted, as well as the runaway candidate VFTS \#16 and the location of the five X-ray hot super-bubbles, identified by Wang \& Helfand (1991).

Chandra Survey "Tarantula-Revealed by X-rays-T-ReX-, PI L. Townsley), and extensively observed at all the wavelengths accessible from the ground, including the VLT-Flames Tarantula Survey (Evans et al. 2011) and the VMC survey in the NIR (Cioni et al. 2011).

High-mass stars in 30 Doradus are so bright that they have been studied for centuries (i.e., de la Caille 1761; Herschel 1847). The high angular resolution and sensitivity of the Hubble Space Telescope (HST) has allowed in-depth studies of dense clusters and rich associations, such as R136, Hodge 301, NGC 2060, and Br73 (Hunter et al. 1995, 1996; Selman et al. 1999; Walborn et al. 1999, 2002; Grebel \& Chu 2000; Brandner et al. 2001; Mignani et al. 2015; Andersen et al. 2009; De Marchi et al. 2011; Sabbi et al. 2012; Selman \& Melnick 2013), whose position relative to the Nebula is shown in Figure 1.

The Hubble Tarantula Treasury Project (HTTP; Cycle 20, HST GO-12939, PI: E. Sabbi) is a photometric survey of the entire nebula from the near-UV to the near-IR at the high sensitivity and angular resolution of $H S T$. HTTP covers approximately $14^{\prime} \times 12^{\prime}$, which, at the distance of the LMC, corresponds to $\sim 210 \times 180 \mathrm{pc}$. Preliminary results from the analysis of half of the observations acquired in the IR filters, as well as a discussion of the main goals of the survey, have been presented in Sabbi et al. (2013).

In this paper, we present the photometric measurements in all eight filters covered by HTTP. The observations (including filter choice, exposure times, and orientation of the various pointings), and the construction of the reference frame are discussed in Section 2. In Section 3 we introduce the photometric package KS2 used to analyze the data. Details of the photometric catalog and artificial star tests are given in Section 4. Color-magnitude diagrams (CMDs) and reddening distributions are presented in Section 5, while in Section 6 we discuss the ages and spatial distribution of the stellar populations found in the Tarantula Nebula. A summary and conclusions are presented in Section 7. 


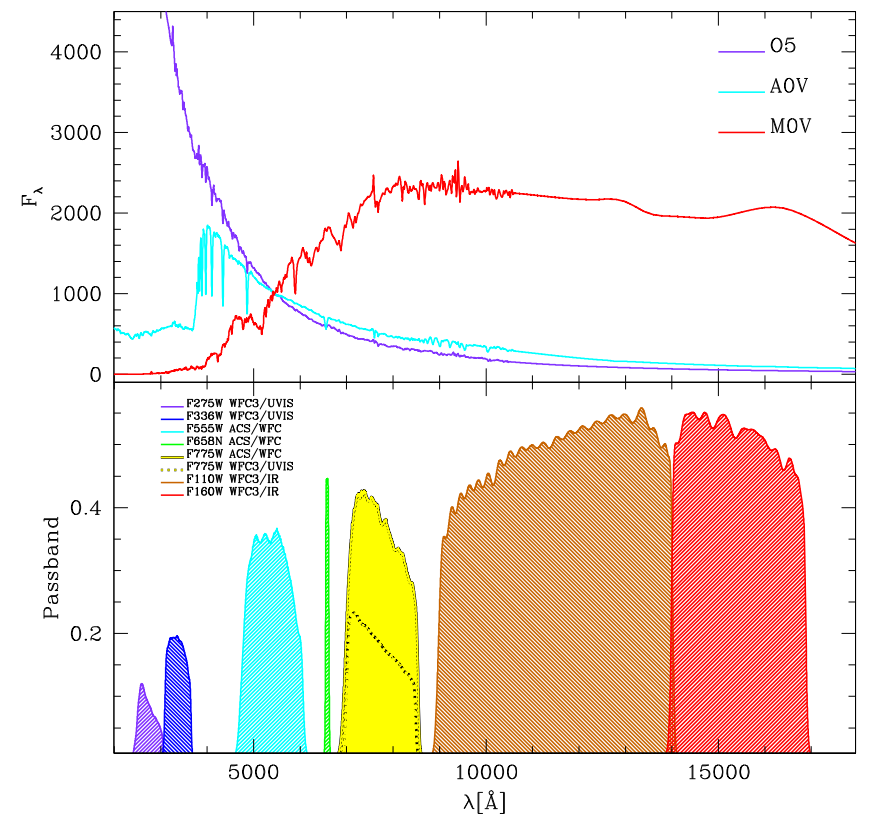

Figure 2. Passbands of the filters used in HTTP as a function of wavelength is shown in the lower Ppanel. The upper panel shows Kurucz 1993 models for an O5 (shown in purple), an A0V (in cyan), and a MOV (in red) stars in the range of wavelengths covered by HTTP.

\section{OBSERVATIONS}

\subsection{The Data}

HTTP was awarded 60 orbits of HST time in cycle 20 to survey the entire Tarantula Nebula, using both the UVIS and the IR channels of the Wide Field Camera 3 (WFC3), and, in parallel, the Wide Filed Channel (WFC) of the Advanced Camera for Surveys (ACS). Each of the UVIS orbits consists of one short (14 s) and two long (697 s each) exposures in the filter F336W followed by two long exposures (697 and $467 \mathrm{~s}$ respectively) in the F275W filter. Each couple of UVIS long exposures were obtained using a two-point dither pattern to enable the rejection of cosmic rays (CRs) and cover the chip gap.

HTTP was built on top of the first epoch of the HST monochromatic survey of 30 Doradus (PI: Lennon, GO-12499) designed to measure proper motions of runaway candidates. That survey used the filter F775W ( Sloan Digital Sky Survey $i$ band) and covered a projected area of $\sim 14^{\prime} \times 12^{\prime}$. The program consisted of a 15 pointing mosaic using WFC3 and ACS in parallel. The orientation angle of the mosaic $\left(\sim 140^{\circ}\right.$ to the north) was chosen to include the very massive runaway candidate VFTS\#16 (Evans et al. 2010; see Figure 1). This data set is described in Sabbi et al. (2013). Preliminary proper motion results, obtained by combining the first epoch data set to archival WFPC2 images, are presented in Platais et al. (2015).

The spectral coverage of HTTP+Lennon's programs is shown in Figure 2, and the observations are summarized in Table 1, including data set names, pointing coordinates, date and time when observations were collected, exposure time in seconds, number of pointings, instrument used, filter, and

\footnotetext{
18 Although they share the same name, the ACS and WFC3 F775W filters differ in terms of color response, PSF, geometric distortion, and pixel scale. Therefore, the two filters have been analyzed independently.
}

central wavelength of the filter. Once combined with Lennon's data, HTTP consists of eight filters ${ }^{18}$ from the near-UV (NUV) to the near-IR (NIR).

Because of the different camera sizes and orientations, the entire field of view is not uniformly covered in all filters. Figure 3 shows for each filter the final mosaic, while the number of exposures contributing to each pixel of each mosaic is shown in Figure 4. Inspection of these two figures shows, for example, that because the F775W survey was obtained using the two cameras in parallels, the F775W ACS filter covers only $\sim 57 \%$ of the total area (panel (e)) of Figure 3), while the UVIS coverage in this filter is $\sim 49 \%$. Because of the small size of the WFC3 detectors compared to ACS, the WFC3 mosaics are affected by gaps, the two most noticeable affecting the F275W (panel (a)) and the F110W (panel (g)) filters. Table 2 reports the fraction of area covered by each filter with respect to the region surveyed by Lennon and collaborators by combining the two ACS and WFC3 F775W images.

Although the WCF3 had been in space for less than four years when the HTTP data was collected, charge transfer efficiency (CTE) losses were already a concern (Bourque \& Kozurina-Platais 2013), especially when the background is low, as is the case for the UV filters (Baggett \& Anderson 2012). To mitigate the effect of the degrading CTE, we increased the background level of the UV exposures by $12 \mathrm{e}^{-}$using post-flash. ${ }^{19}$

While WFC3 was acquiring the UVIS images, ACS was used to collect one short (13s) and four long $(3 \times 640+1 \times$ $337 \mathrm{~s}$ ) exposures in the filter F555W. For the deep F555W exposures, we used a four-point dither pattern, improving the sampling of the point-spread function (PSF). In addition, by smoothing over the spatial variations in the detector response, averaging the flat-field errors, and removing contamination from hot pixels and cosmic rays, the dither pattern allowed us to improve the photometric accuracy of our observations. Because of the important contribution from diffuse emission, the sky background in all ACS images is always higher then $100 \mathrm{e}^{-}$and therefore there was no need to mitigate CTE losses with post-flash.

The IR orbits consist of two long $(2 \times 799 \mathrm{~s})$ exposures in the F160W filter followed by two long (799 and $499 \mathrm{~s}$, respectively) exposures in the F110W filter. At the same time, ACS was used to obtain four long $(3 \times 640+1 \times 399 \mathrm{~s})$ exposures in the F658N filter. In the IR, the large dither steps adopted allowed us to maximize the survey area and to mitigate the effects of persistence (Long et al. 2013a, 2013b).

All ACS exposures were acquired using a gain of $2 \mathrm{e}^{-} / \mathrm{ADU}$, WFC3/UVIS with gain $1.5 \mathrm{e}^{-} / \mathrm{ADU}$, and WFC3/IR with gain $2.5 \mathrm{e}^{-} / \mathrm{ADU}$. The entire data set was processed with the standard Space Telescope Science Institute calibration pipelines CALWF3 version 3.1.2 or CALACS version 8.1.2 to subtract bias level, superbias, and superdark, to apply the flat-field correction and, for the IR observations, the nonlinearity correction and the up-the-ramp fitting. In addition, CALACS applies a pixel-based CTE correction to the flatfielded images. We applied an analogous correction to the WFC3/UVIS data using the stand alone software routine wfc3uv_ctereverse_parallel.F available at the WFC3 webpage. $^{20}$

\footnotetext{
${ }^{19}$ http://www.stsci.edu/hst/wfc3/ins_performance/CTE/ ANDERSON_UVIS_POSTFLASH_EFFICACY.pdf

${ }^{20} \mathrm{http}: / /$ www.stsci.edu/hst/wfc3/tools/cte_tools
} 
Table 1

Log of the Observations

\begin{tabular}{|c|c|c|c|c|c|c|c|c|c|}
\hline Data Set & $\begin{array}{c}\text { R.A. } \\
(2000.0)\end{array}$ & $\begin{array}{c}\text { decl. } \\
(2000.0)\end{array}$ & Obs. Date & Start Time & $\begin{array}{l}\text { Exp. Time } \\
\text { (s) }\end{array}$ & $\begin{array}{c}\# \text { of } \\
\text { Pointings }\end{array}$ & Instrument & Filter & $\begin{array}{c}\text { Central } \\
\text { Wavelength }\end{array}$ \\
\hline \multicolumn{10}{|c|}{ GO-12939 } \\
\hline JBY001010 & 053935.626 & $\begin{array}{llll}-69 & 10 & 04.27\end{array}$ & 2013 Sep 17 & $16: 29: 40$ & 2270.000 & 4 & ACS/WFC & F555W & 5360.755 \\
\hline JBY07B010 & 053910.427 & -691104.56 & 2013 Sep 23 & $21: 29: 47$ & 2270.000 & 4 & ACS/WFC & F555W & 5360.755 \\
\hline JBY07C010 & 053852.450 & $\begin{array}{llll}-69 & 11 & 17.86\end{array}$ & 2013 Sep 10 & 14:01:57 & 2270.000 & 4 & ACS/WFC & F555W & 5360.755 \\
\hline JBY07D010 & 053819.969 & -691257.32 & 2013 Sep 25 & 00:34:19 & 2270.000 & 4 & ACS/WFC & F555W & 5360.755 \\
\hline JBY07E010 & 053753.035 & $\begin{array}{llll}-69 & 13 & 38.70\end{array}$ & 2013 Sep 21 & $21: 53: 23$ & 2270.000 & 4 & ACS/WFC & F555W & 5360.755 \\
\hline JBY07F010 & 053727.388 & -691431.71 & 2013 Sep 24 & $22: 28: 23$ & 2270.000 & 4 & ACS/WFC & F555W & 5360.755 \\
\hline JBY07G010 & 053921.634 & -690726.59 & 2013 Sep 21 & $04: 22: 46$ & 2270.000 & 4 & ACS/WFC & F555W & 5360.755 \\
\hline JBY07H010 & 053855.986 & -690820.43 & 2013 Sep 25 & $22: 23: 46$ & 2270.000 & 4 & ACS/WFC & F555W & 5360.755 \\
\hline JBY07I010 & 053830.339 & -690914.03 & 2013 Sep 21 & 16:06:30 & 2270.000 & 4 & ACS/WFC & F555W & 5360.755 \\
\hline JBY07J010 & 053804.691 & $\begin{array}{llll}-69 & 10 & 07.39\end{array}$ & 2013 Sep 26 & $00: 12: 20$ & 2270.000 & 4 & ACS/WFC & F555W & 5360.755 \\
\hline JBY07K010 & 053745.476 & -691056.15 & 2013 Sep 26 & 14:44:21 & 2270.000 & 4 & ACS/WFC & F555W & 5360.755 \\
\hline JBY07L010 & 053713.395 & -691153.39 & 2013 Sep 26 & $19: 17: 40$ & 2270.000 & 4 & ACS/WFC & F555W & 5360.755 \\
\hline IBY013020 & 053713.569 & -691102.28 & 2013 Apr 06 & $03: 23: 50$ & 1408.000 & 2 & WFC3/UVIS & F336W & 3354.874 \\
\hline IBY013030 & 053723.334 & $\begin{array}{lll}-69 & 11 & 24.44\end{array}$ & 2013 Apr 06 & 03:55:41 & 1164.000 & 2 & WFC3/UVIS & F275W & 2706.949 \\
\hline IBY07M020 & 053739.876 & $\begin{array}{llll}-69 & 10 & 16.78\end{array}$ & 2013 Apr 09 & $20: 13: 33$ & 1408.000 & 2 & WFC3/UVIS & F336W & 3354.874 \\
\hline IBY07M030 & 053749.644 & -691038.84 & 2013 Apr 09 & $20: 45: 24$ & 1164.000 & 2 & WFC3/UVIS & F275W & 2706.949 \\
\hline IBY07N020 & 053803.992 & -690959.17 & 2013 Mar 28 & $09: 22: 59$ & 1408.000 & 2 & WFC3/UVIS & F336W & 3354.874 \\
\hline IBY07N030 & $0538 \quad 14.331$ & -691011.76 & 2013 Mar 28 & $10: 50: 10$ & 1164.000 & 2 & WFC3/UVIS & F275W & 2706.949 \\
\hline IBY07O020 & 053832.399 & -690845.02 & 2013 Apr 09 & $21: 41: 28$ & 1408.000 & 2 & WFC3/UVIS & F336W & 3354.874 \\
\hline IBY07O030 & 053842.170 & -690906.89 & 2013 Apr 09 & $22: 13: 19$ & 1164.000 & 2 & WFC3/UVIS & F275W & 2706.949 \\
\hline IBY07P020 & 053858.614 & -690758.76 & 2013 Apr 09 & 06:00:34 & 1408.000 & 2 & WFC3/UVIS & F336W & 3354.874 \\
\hline IBY07P030 & 053908.386 & -690820.54 & 2013 Apr 09 & 07:19:59 & 1164.000 & 2 & WFC3/UVIS & F275W & 2706.949 \\
\hline IBY07Q020 & 053924.798 & $\begin{array}{llll}-69 & 07 & 12.25\end{array}$ & 2013 Apr 05 & $08: 43: 55$ & 1408.000 & 2 & WFC3/UVIS & F336W & 3354.874 \\
\hline IBY07Q030 & 053934.572 & -690733.95 & 2013 Apr 05 & 09:29:59 & 1164.000 & 2 & WFC3/UVIS & F275W & 2706.949 \\
\hline IBY07R020 & 053725.828 & -691344.30 & 2013 Apr 10 & $10: 33: 36$ & 1408.000 & 2 & WFC3/UVIS & F336W & 3354.874 \\
\hline IBY07R030 & 053735.618 & -691406.41 & 2013 Apr 10 & $12: 02: 32$ & 1164.000 & 2 & WFC3/UVIS & F275W & 2706.949 \\
\hline IBY07S020 & 053752.183 & -691258.68 & 2013 Apr 10 & $21: 56: 22$ & 1408.000 & 2 & WFC3/UVIS & F336W & 3354.874 \\
\hline IBY07S030 & 053801.974 & $\begin{array}{llll}-69 & 13 & 20.70\end{array}$ & 2013 Apr 07 & $22: 28: 13$ & 1164.000 & 2 & WFC3/UVIS & F275W & 2706.949 \\
\hline IBY07T020 & 053818.507 & $\begin{array}{llll}-69 & 12 & 12.80\end{array}$ & 2013 Apr 11 & $01: 20: 34$ & 1408.000 & 2 & WFC3/UVIS & F336W & 3354.874 \\
\hline IBY07T030 & 053828.299 & -691234.73 & 2013 Apr 11 & 02:59:17 & 1164.000 & 2 & WFC3/UVIS & F275W & 2706.949 \\
\hline IBY07U020 & 053844.799 & -691126.68 & 2013 Apr 11 & 20:38:02 & 1408.000 & 2 & WFC3/UVIS & F336W & 3354.874 \\
\hline IBY07U030 & 053854.594 & -691148.51 & 2013 Apr 11 & $21: 45: 19$ & 1164.000 & 2 & WFC3/UVIS & F275W & 2706.949 \\
\hline IBY07V020 & 053911.061 & $\begin{array}{llll}-69 & 10 & 40.31\end{array}$ & 2013 Apr 12 & $00: 47: 54$ & 1408.000 & 2 & WFC3/UVIS & F336W & 3354.874 \\
\hline IBY07V030 & 053920.857 & -691102.05 & 2013 Apr 12 & $02: 17: 42$ & 1164.000 & 2 & WFC3/UVIS & F275W & 2706.949 \\
\hline IBY07W020 & 053937.292 & -690953.68 & 2013 Apr 11 & 23:07:06 & 1408.000 & 2 & WFC3/UVIS & F336W & 3354.874 \\
\hline IBY07W030 & 053947.089 & $\begin{array}{llll}-69 & 10 & 15.33\end{array}$ & 2013 Apr 11 & $23: 38: 57$ & 1164.000 & 2 & WFC3/UVIS & F275W & 2706.949 \\
\hline JBY013010 & 053651.308 & -690525.54 & 2013 Apr 06 & $03: 25: 14$ & 2270.000 & 4 & ACS/WFC & F555W & 5360.755 \\
\hline JBY07M010 & 053717.516 & -690440.25 & 2013 Apr 09 & $20: 14: 57$ & 2270.000 & 4 & ACS/WFC & F555W & 5360.755 \\
\hline JBY07N010 & 053730.966 & -690448.73 & 2013 Mar 28 & $09: 24: 23$ & 2270.000 & 4 & ACS/WFC & F555W & 5360.755 \\
\hline JBY07O010 & 053809.841 & -690308.91 & 2013 Apr 09 & $21: 42: 52$ & 2270.000 & 4 & ACS/WFC & F555W & 5360.755 \\
\hline JBY07P010 & 053835.957 & -690222.87 & 2013 Apr 09 & 06:01:58 & 2270.000 & 4 & ACS/WFC & F555W & 5360.755 \\
\hline JBY07Q010 & 053902.044 & -690136.58 & 2013 Apr 05 & $08: 45: 19$ & 2270.000 & 4 & ACS/WFC & F555W & 5360.755 \\
\hline JBY07R010 & 053703.469 & -690807.65 & 2013 Apr 10 & $10: 35: 00$ & 2270.000 & 4 & ACS/WFC & F555W & 5360.755 \\
\hline JBY07S010 & 053729.724 & -690722.24 & 2013 Apr 07 & $21: 57: 46$ & 2270.000 & 4 & ACS/WFC & F555W & 5360.755 \\
\hline JBY07T010 & 053755.948 & -690636.59 & 2013 Apr 11 & $01: 21: 58$ & 2270.000 & 4 & ACS/WFC & F555W & 5360.755 \\
\hline JBY07U010 & 053822.142 & -690550.68 & 2013 Apr 11 & $20: 39: 26$ & 2270.000 & 4 & ACS/WFC & F555W & 5360.755 \\
\hline JBY07V010 & 053848.305 & -690504.52 & 2013 Apr 11 & $00: 49: 18$ & 2270.000 & 4 & ACS/WFC & F555W & 5360.755 \\
\hline JBY07W010 & $\begin{array}{llll}05 & 39 & 14.438\end{array}$ & -690418.11 & 2013 Apr 11 & 23:08:30 & 2270.000 & 4 & ACS/WFC & F555W & 5360.755 \\
\hline JBY01N010 & 053837.689 & -690138.30 & 2013 May 29 & $17: 22: 56$ & 2220.000 & 4 & ACS/WFC & F658N & 6583.956 \\
\hline JBY01O010 & 053846.440 & -690231.80 & 2013 May 22 & $10: 20: 48$ & 2220.000 & 4 & ACS/WFC & F658N & 6583.956 \\
\hline JBY01P010 & 053851.411 & -690306.40 & 2013 May 29 & $23: 06: 25$ & 2220.000 & 4 & ACS/WFC & F658N & 6583.956 \\
\hline JBY01Q010 & 053928.323 & -690628.59 & 2013 May 30 & $16: 41: 54$ & 2220.000 & 4 & ACS/WFC & F658N & 6583.956 \\
\hline JBY01R010 & 053945.201 & -690805.15 & 2013 May 31 & $12: 12: 18$ & 2220.000 & 4 & ACS/WFC & F658N & 6583.956 \\
\hline JBY01S010 & 053804.811 & -690120.60 & 2013 Jun 01 & $12: 31: 47$ & 2220.000 & 4 & ACS/WFC & F658N & 6583.956 \\
\hline JBY01T010 & 053821.689 & -690257.67 & 2013 Jun 02 & 18:01:35 & 2220.000 & 4 & ACS/WFC & F658N & 6583.956 \\
\hline JBY01U010 & 053831.372 & -690349.02 & 2013 Jun 10 & 14:18:59 & 2220.000 & 4 & ACS/WFC & F658N & 6583.956 \\
\hline JBY01V010 & 053855.445 & -690611.50 & 2013 Jun 03 & $13: 00: 52$ & 2220.000 & 4 & ACS/WFC & F658N & 6583.956 \\
\hline JBY01W010 & 053912.323 & -690748.26 & 2013 Jun 11 & 04:02:08 & 2220.000 & 4 & ACS/WFC & F658N & 6583.956 \\
\hline JBY01X010 & 053929.201 & $\begin{array}{lll}-69 & 09 & 24.92\end{array}$ & 2013 Jun 04 & 11:14:42 & 2220.000 & 4 & ACS/WFC & F658N & 6583.956 \\
\hline
\end{tabular}


Table 1

(Continued)

\begin{tabular}{|c|c|c|c|c|c|c|c|c|c|}
\hline Data Set & $\begin{array}{c}\text { R.A. } \\
(2000.0)\end{array}$ & $\begin{array}{c}\text { decl. } \\
(2000.0)\end{array}$ & Obs. Date & Start Time & $\begin{array}{l}\text { Exp. Time } \\
\text { (s) }\end{array}$ & $\begin{array}{c}\# \text { of } \\
\text { Pointings }\end{array}$ & Instrument & Filter & $\begin{array}{c}\text { Central } \\
\text { Wavelength }\end{array}$ \\
\hline JBY01Y010 & 053748.811 & -690239.78 & 2013 Jun 04 & $16: 22: 00$ & 2220.000 & 4 & ACS/WFC & F658N & 6583.956 \\
\hline JBY01Z010 & 053805.689 & -690416.95 & 2013 Jun 06 & $06: 08: 32$ & 2220.000 & 4 & ACS/WFC & F658N & 6583.956 \\
\hline JBY02A010 & 053822.567 & -690554.01 & 2013 Jun 07 & $14: 33: 07$ & 2220.000 & 4 & ACS/WFC & F658N & 6583.956 \\
\hline JBY02B010 & 053839.445 & -690730.97 & 2013 Jun 07 & $20: 55: 29$ & 2220.000 & 4 & ACS/WFC & F658N & 6583.956 \\
\hline JBY02C010 & 053856.323 & -690907.82 & 2013 Jun 11 & 18:59:39 & 2220.000 & 4 & ACS/WFC & F658N & 6583.956 \\
\hline JBY02D010 & 053913.147 & -691019.49 & 2013 Jun 12 & $17: 16: 09$ & 2220.000 & 4 & ACS/WFC & F658N & 6583.956 \\
\hline JBY069010 & 053819.716 & -690008.84 & 2013 May 28 & $11: 21: 23$ & 2220.000 & 4 & ACS/WFC & F658N & 6583.956 \\
\hline IBY01N020 & 053746.039 & -690519.30 & 2013 May 29 & $17: 21: 51$ & 1298.465 & 2 & WFC3/IR & F110W & 11534.459 \\
\hline IBY01N030 & 053746.377 & -690451.07 & 2013 May 29 & $18: 14: 36$ & 1598.466 & 2 & WFC3/IR & F160W & 15369.176 \\
\hline IBY01O020 & 053801.824 & -690653.35 & 2013 May 22 & $10: 19: 43$ & 1298.465 & 2 & WFC3/IR & F110W & 11534.459 \\
\hline IBY01O030 & 053801.332 & -690625.19 & 2013 May 22 & $12: 45: 56$ & 1598.466 & 2 & WFC3/IR & F160W & 15369.176 \\
\hline IBY01P020 & 053821.522 & -690822.52 & 2013 May 22 & $23: 05: 21$ & 1298.465 & 2 & WFC3/IR & F110W & 11534.459 \\
\hline IBY01P030 & 053819.630 & -690756.10 & 2013 May 29 & $23: 29: 29$ & 1598.466 & 2 & WFC3/IR & F160W & 15369.176 \\
\hline IBY01Q020 & 053836.482 & -691009.59 & 2013 May 30 & $16: 40: 49$ & 1298.465 & 2 & WFC3/IR & F110W & 11534.459 \\
\hline IBY01Q030 & 053836.821 & -690941.36 & 2013 May 30 & $17: 04: 57$ & 1598.466 & 2 & WFC3/IR & F160W & 15369.176 \\
\hline IBY01R020 & 053853.296 & -691146.15 & 2013 May 31 & $12: 11: 13$ & 1298.465 & 2 & WFC3/IR & F110W & 11534.459 \\
\hline IBY01R030 & 053853.636 & -691117.92 & 2013 May 31 & $13: 17: 24$ & 1598.466 & 2 & WFC3/IR & F160W & 15369.176 \\
\hline IBY01S020 & 053713.173 & -690501.60 & 2013 Jun 01 & $12: 30: 42$ & 1298.465 & 2 & WFC3/IR & F110W & 11534.459 \\
\hline IBY01S030 & $0537 \quad 13.511$ & -690433.37 & 2013 Jun 01 & $13: 26: 50$ & 1598.466 & 2 & WFC3/IR & F160W & 15369.176 \\
\hline IBY01T020 & 053729.987 & -690638.67 & 2013 Jun 02 & $18: 00: 30$ & 1298.465 & 2 & WFC3/IR & F110W & 11534.459 \\
\hline IBY01T030 & 053730.326 & -690610.44 & 2013 Jun 02 & $18: 24: 38$ & 1598.466 & 2 & WFC3/IR & F160W & 15369.176 \\
\hline IBY01U020 & 053747.743 & -690815.51 & 2013 Jun 10 & $14: 17: 55$ & 1298.465 & 2 & WFC3/IR & F110W & 11534.459 \\
\hline IBY01U030 & 053747.140 & -690747.41 & 2013 Jun 10 & $15: 12: 03$ & 1598.466 & 2 & WFC3/IR & F160W & 15369.176 \\
\hline IBY01V020 & 053803.616 & -690952.50 & 2013 Jun 03 & $12: 59: 47$ & 1298.465 & 2 & WFC3/IR & F110W & 11534.459 \\
\hline IBY01V030 & 053803.955 & -690924.27 & 2013 Jun 03 & $13: 23: 55$ & 1598.466 & 2 & WFC3/IR & F160W & 15369.176 \\
\hline IBY01W020 & 053820.430 & -691129.26 & 2013 Jun 11 & 04:01:03 & 1298.465 & 2 & WFC3/IR & F110W & 11534.459 \\
\hline IBY01W030 & 053820.770 & -691101.03 & 2013 Jun 11 & $04: 25: 11$ & 1598.466 & 2 & WFC3/IR & F160W & 15369.176 \\
\hline IBY01X020 & 053837.244 & -691305.92 & 2013 Jun 04 & $11: 13: 37$ & 1298.465 & 2 & WFC3/IR & F110W & 11534.459 \\
\hline IBY01X030 & 053837.584 & -691237.69 & 2013 Jun 04 & $11: 37: 45$ & 1598.466 & 2 & WFC3/IR & F160W & 15369.176 \\
\hline IBY01Y020 & 053657.121 & -690620.78 & 2013 Jun 04 & $16: 20: 55$ & 1298.465 & 2 & WFC3/IR & F110W & 11534.459 \\
\hline IBY01Y030 & 053657.459 & -690552.55 & 2013 Jun 04 & $16: 45: 03$ & 1598.466 & 2 & WFC3/IR & F160W & 15369.176 \\
\hline IBY01Z020 & 053713.935 & -690757.95 & 2013 Jun 06 & $06: 07: 27$ & 1298.465 & 2 & WFC3/IR & F110W & 11534.459 \\
\hline IBY01Z030 & 053714.274 & -690729.72 & 2013 Jun 06 & $06: 31: 35$ & 1598.466 & 2 & WFC3/IR & F160W & 15369.176 \\
\hline IBY02A020 & 053730.749 & -690935.01 & 2013 Jun 07 & $14: 32: 02$ & 1298.465 & 2 & WFC3/IR & F110W & 11534.459 \\
\hline IBY02A030 & 053731.088 & -690906.78 & 2013 Jun 07 & $15: 32: 07$ & 1598.466 & 2 & WFC3/IR & F160W & 15369.176 \\
\hline IBY02B020 & 053747.564 & -691111.97 & 2013 Jun 07 & $20: 54: 24$ & 1298.465 & 2 & WFC3/IR & F110W & 11534.459 \\
\hline IBY02B030 & 053747.903 & -691043.74 & 2013 Jun 07 & $21: 54: 11$ & 1598.466 & 2 & WFC3/IR & F160W & 15369.176 \\
\hline IBY02C020 & 053804.378 & -691248.83 & 2013 Jun 11 & $18: 58: 34$ & 1298.465 & 2 & WFC3/IR & F110W & 11534.459 \\
\hline IBY02C030 & 053804.718 & -691220.60 & 2013 Jun 11 & $19: 52: 43$ & 1598.466 & 2 & WFC3/IR & F160W & 15369.176 \\
\hline IBY02D020 & 053824.165 & $-6914 \quad 19.25$ & 2013 Jun 12 & $17: 15: 04$ & 1298.465 & 2 & WFC3/IR & F110W & 11534.459 \\
\hline IBY02D030 & 053824.135 & -691350.97 & 2013 Jun 12 & 18:11:09 & 1598.466 & 2 & WFC3/IR & F160W & 15369.176 \\
\hline IBY069020 & 053728.125 & -690349.84 & 2013 May 28 & $11: 20: 18$ & 1298.465 & 2 & WFC3/IR & F110W & 11534.459 \\
\hline IBY069030 & 053728.462 & -690321.61 & 2013 May 28 & $12: 19: 11$ & 1598.466 & 2 & WFC3/IR & F160W & 15369.176 \\
\hline IBY03A020 & 053900.765 & -690822.47 & $2012 \operatorname{Dec} 12$ & 03:33:41 & 1298.465 & 2 & WFC3/IR & F110W & 11534.459 \\
\hline IBY03A030 & 053900.799 & -690850.76 & 2012 Dec 12 & $03: 57: 49$ & 1598.466 & 2 & WFC3/IR & F160W & 15369.176 \\
\hline IBY03B020 & 053843.186 & -690649.63 & 2012 Dec 12 & $08: 56: 26$ & 1298.465 & 2 & WFC3/IR & F110W & 11534.459 \\
\hline IBY03B030 & 053843.214 & $-6907 \quad 17.91$ & $2012 \operatorname{Dec} 12$ & $09: 54: 40$ & 1598.466 & 2 & WFC3/IR & F160W & 15369.176 \\
\hline IBY03C020 & 053825.648 & -690516.68 & 2012 Dec 12 & 20:06:19 & 1298.465 & 2 & WFC3/IR & F110W & 11534.459 \\
\hline IBY03C030 & 053825.670 & -690544.96 & $2012 \operatorname{Dec} 12$ & $21: 37: 28$ & 1598.466 & 2 & WFC3/IR & F160W & 15369.176 \\
\hline IBY03D020 & 053808.152 & -690343.61 & 2012 Dec 13 & $21: 00: 36$ & 1298.465 & 2 & WFC3/IR & F110W & 11534.459 \\
\hline IBY03D030 & 053808.168 & -690411.90 & $2012 \operatorname{Dec} 13$ & 21:47:08 & 1598.466 & 2 & WFC3/IR & F160W & 15369.176 \\
\hline IBY03E020 & 053750.697 & $\begin{array}{lll}-69 & 02 & 10.44\end{array}$ & 2012 Dec 13 & $04: 19: 38$ & 1298.465 & 2 & WFC3/IR & F110W & 11534.459 \\
\hline IBY03E030 & 053750.706 & -690238.72 & 2012 Dec 13 & $05: 16: 41$ & 1598.466 & 2 & WFC3/IR & F160W & 15369.176 \\
\hline IBY03F020 & 053933.742 & -690831.86 & 2012 Dec 14 & $14: 57: 02$ & 1298.465 & 2 & WFC3/IR & F110W & 11534.459 \\
\hline IBY03F030 & 053933.788 & -690900.15 & $2012 \operatorname{Dec} 14$ & $15: 21: 10$ & 1598.466 & 2 & WFC3/IR & F160W & 15369.176 \\
\hline IBY03G020 & 053916.122 & -690659.23 & 2012 Dec 13 & 13:07:40 & 1298.465 & 2 & WFC3/IR & F110W & 11534.459 \\
\hline IBY03G030 & 053916.162 & -690727.52 & 2012 Dec 13 & $13: 31: 48$ & 1598.466 & 2 & WFC3/IR & F160W & 15369.176 \\
\hline IBY03H020 & 053858.543 & -690526.49 & 2012 Dec 17 & $15: 00: 52$ & 1298.465 & 2 & WFC3/IR & F110W & 11534.459 \\
\hline IBY03H030 & 053858.577 & -690554.77 & 2012 Dec 17 & $16: 13: 51$ & 1598.466 & 2 & WFC3/IR & F160W & 15369.176 \\
\hline IBY03I020 & 053841.006 & -690353.63 & 2012 Dec 14 & $09: 43: 38$ & 1298.465 & 2 & WFC3/IR & F110W & 11534.459 \\
\hline
\end{tabular}


Table 1

(Continued)

\begin{tabular}{|c|c|c|c|c|c|c|c|c|c|}
\hline Data Set & $\begin{array}{c}\text { R.A. } \\
(2000.0)\end{array}$ & $\begin{array}{c}\text { decl. } \\
(2000.0)\end{array}$ & Obs. Date & Start Time & $\begin{array}{l}\text { Exp. Time } \\
\text { (s) }\end{array}$ & $\begin{array}{c}\text { \# of } \\
\text { Pointings }\end{array}$ & Instrument & Filter & $\begin{array}{c}\text { Central } \\
\text { Wavelength }\end{array}$ \\
\hline IBY03I030 & 053841.033 & -690421.92 & 2012 Dec 14 & 10:07:46 & 1598.466 & 2 & WFC3/IR & F160W & 15369.176 \\
\hline IBY03J020 & 053823.510 & -690220.67 & 2012 Dec 15 & 07:09:47 & 1298.465 & 2 & WFC3/IR & F110W & 11534.459 \\
\hline IBY03J030 & 053823.531 & -690248.95 & 2012 Dec 15 & 08:01:28 & 1598.466 & 2 & WFC3/IR & F160W & 15369.176 \\
\hline IBY03K020 & 053806.055 & -690047.59 & 2012 Dec 16 & $07: 20: 27$ & 1298.465 & 2 & WFC3/IR & F110W & 11534.459 \\
\hline IBY03K030 & 053806.070 & -690115.87 & 2012 Dec 16 & 08:09:42 & 1598.466 & 2 & WFC3/IR & F160W & 15369.176 \\
\hline IBY03L020 & 053949.066 & -690708.44 & 2012 Dec 16 & $18: 16: 19$ & 1298.465 & 2 & WFC3/IR & F110W & 11534.459 \\
\hline IBY03L030 & 053949.117 & -690736.72 & 2012 Dec 16 & $19: 38: 33$ & 1598.466 & 2 & WFC3/IR & F160W & 15369.176 \\
\hline IBY03M020 & 053931.753 & -690511.27 & 2012 Dec 09 & 03:50:39 & 1298.465 & 2 & WFC3/IR & F110W & 11534.459 \\
\hline IBY03M030 & 053932.378 & -690539.36 & 2012 Dec 09 & $04: 14: 47$ & 1598.466 & 2 & WFC3/IR & F160W & 15369.176 \\
\hline IBY03N020 & 053913.868 & -690403.26 & 2012 Dec 18 & $21: 19: 16$ & 1298.465 & 2 & WFC3/IR & F110W & 11534.459 \\
\hline IBY03N030 & 053913.907 & -690431.55 & 2012 Dec 18 & 21:59:59 & 1598.466 & 2 & WFC3/IR & F160W & 15369.176 \\
\hline IBY03O020 & 053856.331 & -690230.51 & $2012 \operatorname{Dec} 17$ & $08: 37: 55$ & 1298.465 & 2 & WFC3/IR & F110W & 11534.459 \\
\hline IBY03O030 & 053856.364 & -690258.79 & 2012 Dec 17 & $09: 23: 49$ & 1598.466 & 2 & WFC3/IR & F160W & 15369.176 \\
\hline IBY03P020 & 053838.836 & -690057.64 & 2012 Dec 17 & $23: 43: 25$ & 1298.465 & 2 & WFC3/IR & F110W & 11534.459 \\
\hline IBY03P030 & 053838.863 & -690125.92 & 2012 Dec 18 & 00:07:33 & 1598.466 & 2 & WFC3/IR & F160W & 15369.176 \\
\hline IBY03Q020 & 053821.381 & -685924.66 & 2012 Dec 18 & $06: 58: 18$ & 1298.465 & 2 & WFC3/IR & F110W & 11534.459 \\
\hline IBY03Q030 & 053821.402 & -685952.94 & 2012 Dec 18 & $07: 40: 52$ & 1598.466 & 2 & WFC3/IR & F160W & 15369.176 \\
\hline IBY087020 & 053918.385 & -690955.20 & 2012 Dec 19 & 03:38:09 & 1298.465 & 2 & WFC3/IR & F110W & 11534.459 \\
\hline IBY087030 & 053918.426 & $\begin{array}{llll}-69 & 10 & 23.48\end{array}$ & 2012 Dec 19 & $04: 21: 42$ & 1598.466 & 2 & WFC3/IR & F160W & 15369.176 \\
\hline JBY03A010 & 053811.741 & -691221.56 & 2012 Dec 12 & $03: 34: 45$ & 2220.000 & 4 & ACS/WFC & F658N & 6583.956 \\
\hline JBY03B010 & 053754.166 & -691048.40 & 2012 Dec 12 & $08: 57: 30$ & 2220.000 & 4 & ACS/WFC & F658N & 6583.956 \\
\hline JBY03C010 & 053736.634 & -690915.14 & 2012 Dec 12 & $20: 07: 23$ & 2220.000 & 4 & ACS/WFC & F658N & 6583.956 \\
\hline JBY03D010 & 053719.142 & -690741.76 & 2012 Dec 13 & 21:01:40 & 2220.000 & 4 & ACS/WFC & F658N & 6583.956 \\
\hline JBY03E010 & 053701.693 & -690608.28 & 2012 Dec 13 & $04: 20: 42$ & 2220.000 & 4 & ACS/WFC & F658N & 6583.956 \\
\hline JBY03F010 & 053844.812 & -691231.53 & 2012 Dec 14 & 14:58:06 & 2220.000 & 4 & ACS/WFC & F658N & 6583.956 \\
\hline JBY03G010 & 053827.196 & -691058.59 & 2012 Dec 13 & $13: 08: 44$ & 2220.000 & 4 & ACS/WFC & F658N & 6583.956 \\
\hline JBY03H010 & 053809.622 & -690925.54 & 2012 Dec 17 & $15: 01: 56$ & 2220.000 & 4 & ACS/WFC & F658N & 6583.956 \\
\hline JBY03I010 & 053752.090 & -690752.37 & 2012 Dec 14 & $09: 44: 42$ & 2220.000 & 4 & ACS/WFC & F658N & 6583.956 \\
\hline JBY03J010 & 053734.599 & -690619.09 & 2012 Dec 15 & $07: 10: 51$ & 2220.000 & 4 & ACS/WFC & F658N & 6583.956 \\
\hline JBY03K010 & 053717.149 & -690445.70 & 2012 Dec 16 & $07: 21: 31$ & 2220.000 & 4 & ACS/WFC & F658N & 6583.956 \\
\hline JBY03L010 & 053900.235 & -691108.38 & 2012 Dec 16 & $18: 17: 23$ & 2220.000 & 4 & ACS/WFC & F658N & 6583.956 \\
\hline JBY03M010 & 053848.157 & -690938.14 & 2012 Dec 09 & $03: 51: 43$ & 2220.000 & 4 & ACS/WFC & F658N & 6583.956 \\
\hline JBY03N010 & 053825.046 & -690802.58 & 2012 Dec 18 & $21: 20: 20$ & 2220.000 & 4 & ACS/WFC & F658N & 6583.956 \\
\hline JBY03O010 & 053807.513 & -690629.51 & 2012 Dec 17 & 08:38:59 & 2220.000 & 4 & ACS/WFC & F658N & 6583.956 \\
\hline JBY03P010 & 053750.022 & -690456.33 & 2012 Dec 17 & $23: 44: 29$ & 2220.000 & 4 & ACS/WFC & F658N & 6583.956 \\
\hline JBY03Q010 & 053732.573 & -690323.04 & 2012 Dec 18 & $06: 59: 22$ & 2220.000 & 4 & ACS/WFC & F658N & 6583.956 \\
\hline JBY087010 & 053829.357 & -691354.60 & 2012 Dec 19 & $03: 39: 13$ & 2220.000 & 4 & ACS/WFC & F658N & 6583.956 \\
\hline IBY001020 & 053906.435 & -690443.03 & 2013 Sep 17 & $16: 28: 16$ & 1408.000 & 2 & WFC3/UVIS & F336W & 3354.874 \\
\hline IBY001030 & 053856.323 & -690426.74 & 2013 Sep 17 & $17: 52: 45$ & 1164.000 & 2 & WFC3/UVIS & F275W & 2706.949 \\
\hline IBY07B020 & 053841.214 & $\begin{array}{lll}-69 & 05 & 43.32\end{array}$ & 2013 Sep 23 & $21: 28: 23$ & 1408.000 & 2 & WFC3/UVIS & F336W & 3354.874 \\
\hline IBY07B030 & 053831.094 & -690527.03 & 2013 Sep 23 & $22: 23: 09$ & 1164.000 & 2 & WFC3/UVIS & F275W & 2706.949 \\
\hline IBY07C020 & 053807.740 & -690652.21 & 2013 Sep 10 & $14: 00: 33$ & 1408.000 & 2 & WFC3/UVIS & F336W & 3354.874 \\
\hline IBY07C030 & 053757.163 & -690651.48 & 2013 Sep 10 & $15: 16: 40$ & 1164.000 & 2 & WFC3/UVIS & F275W & 2706.949 \\
\hline IBY07D020 & 053750.714 & -690736.08 & 2013 Sep 25 & $00: 32: 55$ & 1408.000 & 2 & WFC3/UVIS & F336W & 3354.874 \\
\hline IBY07D030 & 053740.580 & -690719.79 & 2013 Sep 25 & 01:04:46 & 1164.000 & 2 & WFC3/UVIS & F275W & 2706.949 \\
\hline IBY07E020 & 053723.765 & -690817.46 & 2013 Sep 21 & $21: 51: 59$ & 1408.000 & 2 & WFC3/UVIS & F336W & 3354.874 \\
\hline IBY07E030 & 053713.625 & -690801.17 & 2013 Sep 21 & $23: 24: 21$ & 1164.000 & 2 & WFC3/UVIS & F275W & 2706.949 \\
\hline IBY07F020 & 053658.097 & -690910.47 & 2013 Sep 24 & $22: 26: 59$ & 1408.000 & 2 & WFC3/UVIS & F336W & 3354.874 \\
\hline IBY07F030 & 053647.950 & -690854.18 & 2013 Sep 24 & 23:08:11 & 1164.000 & 2 & WFC3/UVIS & F275W & 2706.949 \\
\hline IBY07G020 & 053852.502 & -690205.35 & 2013 Sep 21 & $04: 21: 22$ & 1408.000 & 2 & WFC3/UVIS & F336W & 3354.874 \\
\hline IBY07G030 & 053842.410 & -690149.06 & 2013 Sep 21 & $04: 53: 13$ & 1164.000 & 2 & WFC3/UVIS & F275W & 2706.949 \\
\hline IBY07H020 & 053826.834 & -690259.19 & 2013 Sep 25 & $22: 22: 22$ & 1408.000 & 2 & WFC3/UVIS & F336W & 3354.874 \\
\hline IBY07H030 & $0538 \quad 16.735$ & -690242.90 & 2013 Sep 25 & $23: 00: 17$ & 1164.000 & 2 & WFC3/UVIS & $\mathrm{F} 275 \mathrm{~W}$ & 2706.949 \\
\hline IBY07I020 & 053801.167 & -690352.79 & 2013 Sep 21 & 16:05:06 & 1408.000 & 2 & WFC3/UVIS & F336W & 3354.874 \\
\hline IBY07I030 & 053751.061 & -690336.50 & 2013 Sep 21 & $17: 32: 17$ & 1164.000 & 2 & WFC3/UVIS & F275W & 2706.949 \\
\hline IBY07J020 & 053735.499 & -690446.15 & 2013 Sep 26 & $00: 10: 56$ & 1408.000 & 2 & WFC3/UVIS & F336W & 3354.874 \\
\hline IBY07J030 & 053725.386 & -690429.86 & 2013 Sep 26 & 00:49:39 & 1164.000 & 2 & WFC3/UVIS & F275W & 2706.949 \\
\hline IBY07K020 & 053710.123 & -690553.03 & 2013 Sep 26 & $14: 42: 57$ & 1408.000 & 2 & WFC3/UVIS & F336W & 3354.874 \\
\hline IBY07K030 & 053659.739 & -690542.49 & 2013 Sep 26 & $15: 38: 36$ & 1164.000 & 2 & WFC3/UVIS & F275W & 2706.949 \\
\hline IBY07L020 & 053644.164 & -690632.15 & 2013 Sep 21 & 19:16:16 & 1408.000 & 2 & WFC3/UVIS & F336W & 3354.874 \\
\hline
\end{tabular}


Table 1

(Continued)

\begin{tabular}{|c|c|c|c|c|c|c|c|c|c|}
\hline Data Set & $\begin{array}{c}\text { R.A. } \\
(2000.0)\end{array}$ & $\begin{array}{c}\text { decl. } \\
(2000.0)\end{array}$ & Obs. Date & Start Time & $\begin{array}{l}\text { Exp. Time } \\
\text { (s) }\end{array}$ & $\begin{array}{c}\text { \# of } \\
\text { Pointings }\end{array}$ & Instrument & Filter & $\begin{array}{c}\text { Central } \\
\text { Wavelength }\end{array}$ \\
\hline IBY07L030 & 053634.037 & -690615.86 & 2013 Sep 21 & $20: 14: 00$ & 1164.000 & 2 & WFC3/UVIS & F275W & 2706.949 \\
\hline IBSF01020 & 053901.607 & -690307.51 & 2011 Oct 03 & $17: 32: 38$ & 2639.000 & 4 & WFC3/UVIS & F775W & 7647.629 \\
\hline IBSF03020 & 053829.314 & $-6905 \quad 10.83$ & 2011 Oct 04 & $14: 18: 11$ & 2639.000 & 4 & WFC3/UVIS & F775W & 7647.629 \\
\hline IBSF04020 & $0538 \quad 11.082$ & -690637.57 & 2011 Oct 08 & $13: 09: 23$ & 2639.000 & 4 & WFC3/UVIS & F775W & 7647.629 \\
\hline IBSF05020 & 053756.920 & -690713.77 & 2011 Oct 04 & $17: 16: 27$ & 2639.000 & 4 & WFC3/UVIS & F775W & 7647.629 \\
\hline IBSF09020 & 053842.710 & -690045.31 & 2011 Oct 05 & $23: 50: 49$ & 2639.000 & 4 & WFC3/UVIS & F775W & 7647.629 \\
\hline IBSF10020 & 053826.590 & -690146.90 & 2011 Oct 06 & $03: 15: 22$ & 2639.000 & 4 & WFC3/UVIS & F775W & 7647.629 \\
\hline IBSF11020 & 053810.445 & -690248.40 & 2011 Oct 06 & $12: 36: 51$ & 2639.000 & 4 & WFC3/UVIS & F775W & 7647.629 \\
\hline IBSF12020 & 053754.275 & -690349.81 & 2011 Oct 06 & $15: 21: 17$ & 2639.000 & 4 & WFC3/UVIS & F775W & 7647.629 \\
\hline IBSF13020* & 053739.196 & -690444.99 & 2011 Oct 06 & $18: 46: 44$ & 35.000 & 1 & WFC3/UVIS & F775W & 7647.629 \\
\hline JBSF01010 & 053938.121 & -690806.41 & 2011 Oct 03 & $17: 33: 43$ & 2329.000 & 4 & ACS/WFC & F775W & 7693.671 \\
\hline JBSF02010 & 053921.954 & -690908.33 & 2011 Oct 03 & $23: 57: 12$ & 2329.000 & 4 & ACS/WFC & F775W & 7693.671 \\
\hline JBSF03010 & 053905.762 & -691010.16 & 2011 Oct 04 & $14: 19: 16$ & 2329.000 & 4 & ACS/WFC & F775W & 7693.671 \\
\hline JBSF04010 & 053837.186 & -691206.39 & 2011 Oct 08 & $13: 10: 28$ & 2329.000 & 4 & ACS/WFC & F775W & 7693.671 \\
\hline JBSF05010 & 053833.301 & $-6912 \quad 13.52$ & 2011 Oct 04 & $17: 17: 32$ & 2329.000 & 4 & ACS/WFC & F775W & 7693.671 \\
\hline JBSF06010 & 053801.117 & -691337.45 & 2011 Oct 05 & 09:29:08 & 2329.000 & 4 & ACS/WFC & F775W & 7693.671 \\
\hline JBSF07010 & 053800.738 & -691416.51 & 2011 Oct 05 & 19:04:12 & 2329.000 & 4 & ACS/WFC & F775W & 7693.671 \\
\hline JBSF09010 & 053919.085 & -690544.46 & 2011 Oct 05 & $23: 51: 54$ & 2329.000 & 4 & ACS/WFC & F775W & 7693.671 \\
\hline JBSF10010 & 053902.933 & -690646.27 & 2011 Oct 06 & $03: 16: 27$ & 2329.000 & 4 & ACS/WFC & F775W & 7693.671 \\
\hline JBSF11010 & 053846.755 & -690747.98 & 2011 Oct 06 & $12: 37: 56$ & 2329.000 & 4 & ACS/WFC & F775W & 7693.671 \\
\hline JBSF12010 & 053830.552 & -690849.60 & 2011 Oct 06 & $15: 22: 22$ & 2329.000 & 4 & ACS/WFC & F775W & 7693.671 \\
\hline JBSF13010* & 053805.129 & $-69 \quad 1014.12$ & 2011 Oct 06 & $18: 47: 49$ & 32.000 & 1 & ACS/WFC & F775W & 7693.671 \\
\hline
\end{tabular}

Note. Items marked with * have been discarded because they have been degraded by the loss of the guide stars by the Fine Guidance Sensors.

\subsection{The Reference Frame}

The analysis of the entire HTTP data set has been carried out directly on the pipeline-processed CTE corrected_flc images. Because IR detectors are not affected by CTE losses, the IR analysis was carried on the flt images.

Compared to drizzled (either_drz or_drc) images, _flt and flc images have the advantage of not being resampled and therefore they are the most direct representation of the astronomical scene. On the other hand, these images are still affected by geometric distortion. Thus, the first step in reducing the HTTP data set was to create a distortion-free reference frame and relate the astrometry and photometry of each exposure to this frame. We chose the F775W filters to build the reference frame because, compared, for example, to F555W or F658N, the contribution from the ionized gas in these images is limited, and they are among the deepest exposures and have much higher spatial resolution than the NIR data.

To create the reference frame, we began by running the publicly available one-pass photometry routine img $2 x y m$ (Anderson \& King 2006) on each exposure. We used this routine and a library of empirical PSFs to find and measure all the sources that were brighter than 100 counts in a 3 pixel aperture and had no brighter neighbors within a 5 pixel radius. The 2MASS catalog (Skrutskie et al. 2006) was used to construct a single reference frame that has a pixel scale of 40 mas/pixel (matching that of the WFC3/UVIS camera). The final reference frame measures 32,000 pixels along the $X$ axis and 28,000 pixels along the $Y$ axis to allow the mapping of all pixels covered by the various filters and at the same time minimize the total number of empty pixels in each mosaic.

In doing so, we were able to identify 110,000 bright, isolated, unsaturated stars that could be measured in three or more deep exposures. Since the 2MASS positions are good to only 50 mas, we improved the internal quality of the reference frame by iterating between solving for an average position of each star in the frame and using these average positions to improve the transformation from each exposure into the frame. Within a few iterations, the average positions converged with rms residuals of less than 0.01 pixel.

Once the final reference list was constructed from the bright stars in the $\mathrm{F} 775 \mathrm{~W}$ exposures, we ran the one-pass routine on all the exposures (short and deep for all filters) and crossidentified stars to determine the transformation from each image into the reference frame. The WFC3/IR images were also mapped to this frame with the WFC3/UVIS pixel scale. 


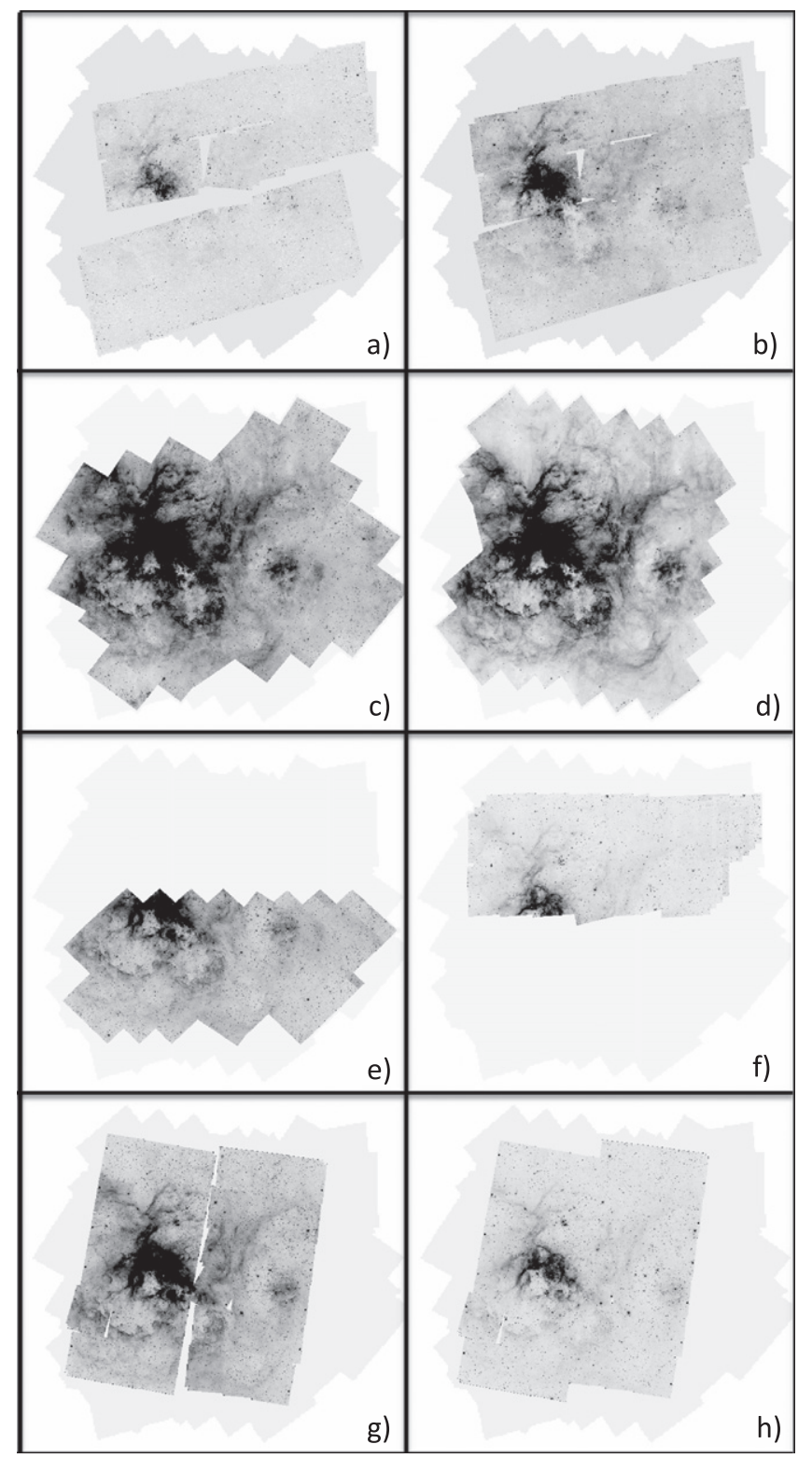

Figure 3. Eight mosaics of the region covered by the HTTP survey in the filters

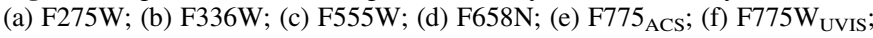
(g) F110W; and (h) F160W. The faint light gray area around the mosaics shows the total area covered by the survey when all the filters are considered.

\section{KS2: A MULTI-PURPOSE FINDING AND PHOTOMETRY ROUTINE}

The one-pass routine used above finds almost all of the bright stars in a field, but it is not designed to find faint objects, since these often require multiple detections in multiple exposures to be found and well measured. To recover these sources, we used KS2, an evolution of the program used to measure the ACS Globular Cluster Treasury Survey (GO10775; see Anderson et al. 2008). A detailed description of KS2 will be presented in J. Anderson et al. (2016, in preparation). In this section, we describe the aspects of KS2 that were used for the reduction of the HTTP data set and to run the artificial star tests.

KS2 requires the transformations from each exposure into the reference frame, the library of PSFs for the relevant filters,

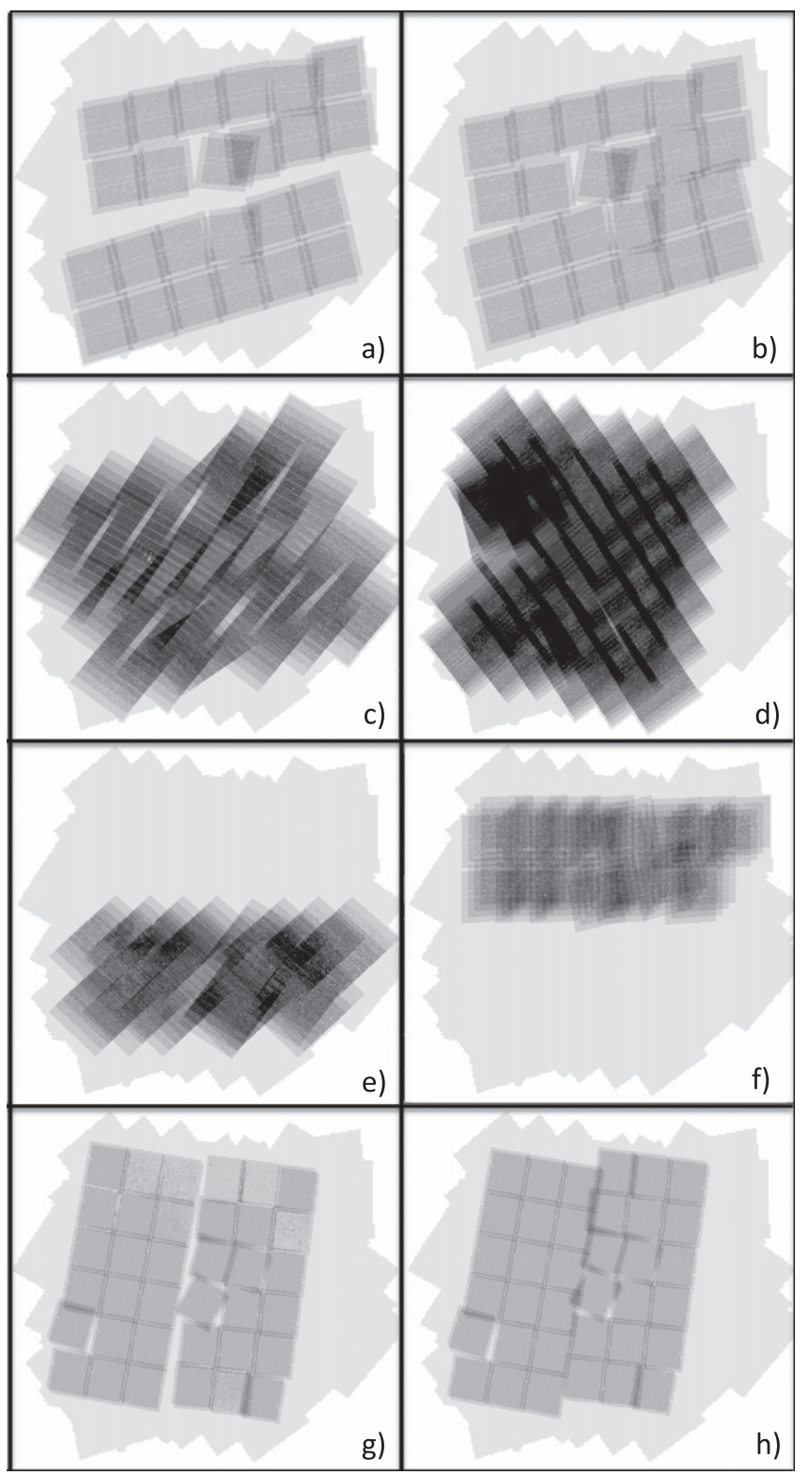

Figure 4. Coverage maps of the eight mosaics shown in Figure 3. The darker the image, the greater the number of overlapping images.

Table 2

Field of View Covered by Each Filter with the Respect to the Two WFC3 and ACS F775W Filters Combined Together

\begin{tabular}{|c|c|}
\hline Filter & Percentage \\
\hline F $775 W_{\text {combined }}$ & $100 \%$ \\
\hline F555W & $89 \%$ \\
\hline F658N & $85 \%$ \\
\hline $\mathrm{F} 275 \mathrm{~W}$ & $82 \%$ \\
\hline F336W & $79 \%$ \\
\hline F110W & $76 \%$ \\
\hline F160W & $75 \%$ \\
\hline $\mathrm{F} 75 \mathrm{~W}_{\mathrm{ACS}}$ & $57 \%$ \\
\hline F775 $\mathrm{W}_{\text {UVIS }}$ & $49 \%$ \\
\hline
\end{tabular}

and a list of the bright stars that are likely to be saturated in many of the frames. KS2 can analyze multiple HST instruments and can process up to 15 filters and hundreds of exposures at the same time. 
KS2 constructs a master catalog of all the sources by going through the reference frame in tiles that are $125 \times 125$ pixels in size. For each tile it makes a list of all exposures that cover it and extracts the relevant raster from each exposure. It executes several finding passes to identify stars that satisfy various criteria such as isolation within 5 pixels, signal-to-noise ratio $(\mathrm{S} / \mathrm{N})$, quality-of-fit, or number of coincident peaks in multiple frames. After each finding pass, the stars are measured and the PSF is used to subtract them from each individual exposure. In addition to the subtraction, the routine also makes a mask for each exposure that allows us to verify what kind of PSF artifacts might be present, either related to the diffraction spikes or poor subtraction. Sources detected in following passes have to stand out above this mask to be included in the catalog.

KS2 is run on the individual_flt and flc exposures, eliminating the need to alter the information present in the images by resampling them with, e.g., drizzle. This approach allows us to determine an independent estimate of each star's flux in each exposure to give us an estimate of measurement errors, time variability, etc. In performing the photometric analysis, KS2 applies a pixel area map correction, thus taking into account the fact that in an image the area of sky covered by different pixels varies as a function of their position.

We chose to use only the F775W, F110W, and F160W exposures to find stars because in these filters both the bluest and reddest stars have the highest $\mathrm{S} / \mathrm{N}$ and the contribution of the ionized gas is not as relevant as in the F555W and F658N filters, reducing the possibility to detect spurious sources, for example, along sharp filaments of gas (Figure 5).

Different approaches are needed to measure different stars. Bright stars are best measured by fitting the PSF to the flt/ _flc pixels to solve for position and flux. For simplicity, we will call the simultaneous fitting of position and flux "method \#1." Faint stars often do not have enough signal to allow their positions to be measured well in individual exposures, so it is best to determine an average position from all the exposures, and then fit each exposure's pixels with the PSF solving only for the flux. Hereafter, we will refer to this method as \#2.

We used both "methods" on all stars and reported in the final catalog the photometry that is most appropriate for each star's signal-to-noise. Figure 6 shows the comparison between the two photometric measurements. The two methods are in very good agreement in the brighter 5-6 mag, but at fainter magnitudes method \#1 tends to underestimate the magnitude of the measured sources.

For the ACS/WFC and WFC3/UVIS exposures, our aperture is the $5 \times 5$ pixel around the star's central pixel, while for WFC $3 / \mathrm{IR}$ it is the $3 \times 3$ pixel, on account of the detector's severely undersampled nature. In method \#1, a robust sky was determined in the source-subtracted image using an annulus between 4 and 8 pixels radii, while in method \#2, we used a smaller annulus (between 3 and 7 pixels). In all cases, the PSF was fit to the selected neighbor-subtracted pixels using a leastsquares approach that took into account all relevant sources of noise, such as Poisson noise, a $\sim 1 \%$ error in the PSF model, error in the sky determination, etc.

When possible, we recovered the information related to saturated stars from the short exposures. However, many of the known OB stars in the region are so bright to be saturated even in those images. In these cases, we measured the fluxes by taking advantage of the fact that the CCD blooming process does not destroy electrons but simply displaces them along the

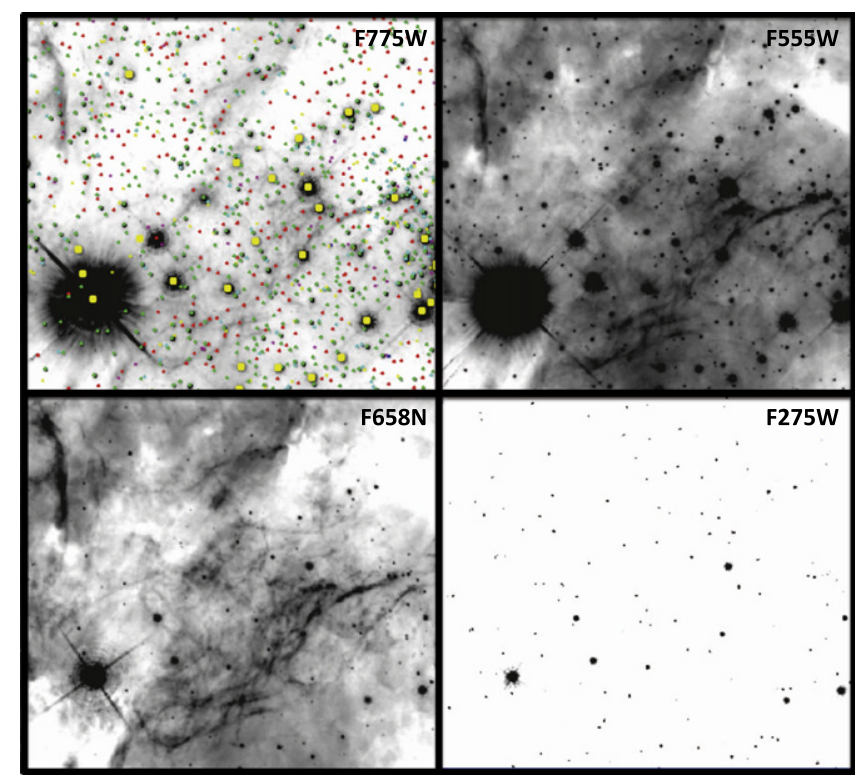

Figure 5. Detail of 30 Doradus in the filters: ACS-F775W (upper left panel), F555W (upper right panel), F658N (lowe left panel), and F275W (lower right panel). Stars detected by the finding algorithm are marked in the F775W image. Different colors correspond to different detection passes.

columns (Gilliland 2004; Gilliland et al. 2010). In particular, we started measuring the flux within a $5 \times 5$ pixel aperture and additionally identify every pixel that is part of the contiguous distribution centered on the target star, as well as any pixel that is next to any saturated pixel in the contiguous distribution. We then added the total amount of flux over the sky in these pixels and determined the fraction of the PSF to which this aperture corresponds. The total flux of the star was then determined by dividing the observed amount of light by the fraction of the star's flux that should have landed in these pixels.

This process suffers from some flat-fielding errors since it is impossible to know which pixel each electron landed in; however, these are small errors and comparison of fluxes measured for stars that were saturated in the deep exposures and those unsaturated in the short exposures indicates this procedure is generally accurate to better than $5 \%$. Figure 7 shows the difference in magnitude for the stars that have been observed with both the F775W filters as a function of magnitude. Above saturation the average difference between the two filters is $m_{\mathrm{F} 775 \mathrm{~W}_{\mathrm{ACS}}}-m_{\mathrm{F} 775 \mathrm{~W}_{\mathrm{UVIS}}}=-0.016 \pm 0.028$.

The output of KS2 provides a wealth of information to help us evaluate the quality of the photometry. The program creates a stack image for each analyzed filter, allowing us to verify that each frame was properly matched. The stacked images for all the HTTP filters are shown in Figure 3. In addition, KS2 provides a coverage map for each filter (shown in Figure 4), which is useful to verify how many images contributed to each pixel in a mosaic. In addition, the code creates stellarsubtracted images to validate that all the point sources have been identified, as well as saturation masks, which is useful to verify where the saturated pixels are and the diffraction spikes. A portion of the saturation mask derived for the filter $\mathrm{F} 555 \mathrm{~W}$ is shown in Figure 8 as an example.

For both the photometric methods described above, KS2 creates a separate photometric catalog. Each catalog includes each source's average flux, the rms of the independent 


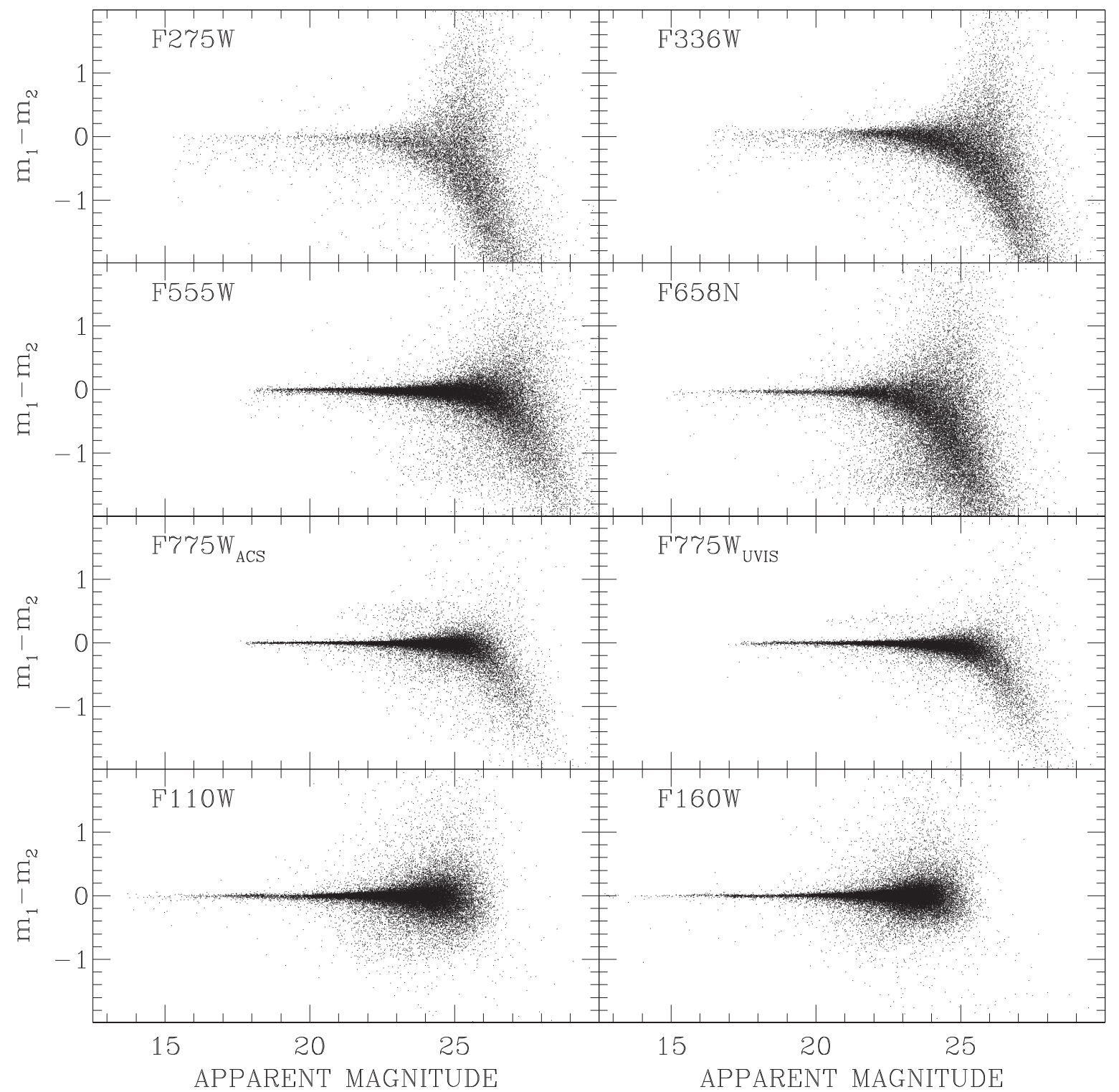

Figure 6. Comparison between the two photometry methods as a function of the apparent magnitude for the filters F275W, F336W, F555W, F658N, ACS/F775W, UVIS/F775W, F110W, and F160W. In each plot only 5\% of the sources are shown.

estimates from the contributing exposures, the number of images where the star could have been found, the number in which it was measured well, the finding pass in which it was found, a metric of the quality of the fit of the PSF to its pixels $\left(Q_{\mathrm{fit}}\right)$, etc. Figures 9 and 10 show the distributions of the photometric errors and quality of the fit as a function of magnitudes for each of the 8 filters in the HTTP data set. Both plots show that KS2 is very efficient in detecting very faint sources $(m>28)$; however, when the $\mathrm{S} / \mathrm{N}$ is low the quality of the fit becomes very poor.

\section{THE PHOTOMETRIC CATALOG}

As mentioned before, KS2 measures the flux of all the stars found in different ways. In the final catalog we used the flux measured with method \#1 if a star was found within the first detection pass. In all other cases we used the flux derived from method \#2.

Fluxes were converted into instrumental magnitudes and then calibrated to the VEGAmag photometric system using the zero points listed on the STScI Web site. ${ }^{21}$ The zero points are derived for images combined using "astrodrizzle" and cannot be directly applied to _flt/_flc-based catalogs.

To calibrate our data to VEGAmag, we selected several isolated bright stars in the drizzle images and measured their magnitudes using a 0.5 aperture photometry for ACS data and 0 ". 4 for WFC3. We used the average difference between the KS2 instrumental magnitudes and the calibrated magnitudes obtained from the drizzled images to put our catalog into the VEGAmag photometric system.

Figure 7 shows the difference in magnitude between sources that have been observed in both the F775W filters as a function of magnitude in the ACS $/ \mathrm{F} 775 \mathrm{~W}$ filter. After the calibration in VEGAmag, the average difference between the two photometric catalogs is 0.014 . Such a difference can be ascribed to a combination of color terms (for red stars the difference drops to $0.006)$ and uncertainties in the aperture correction.

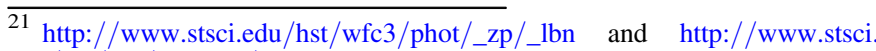
edu/hst/acs/analysis/zeropoints
} 


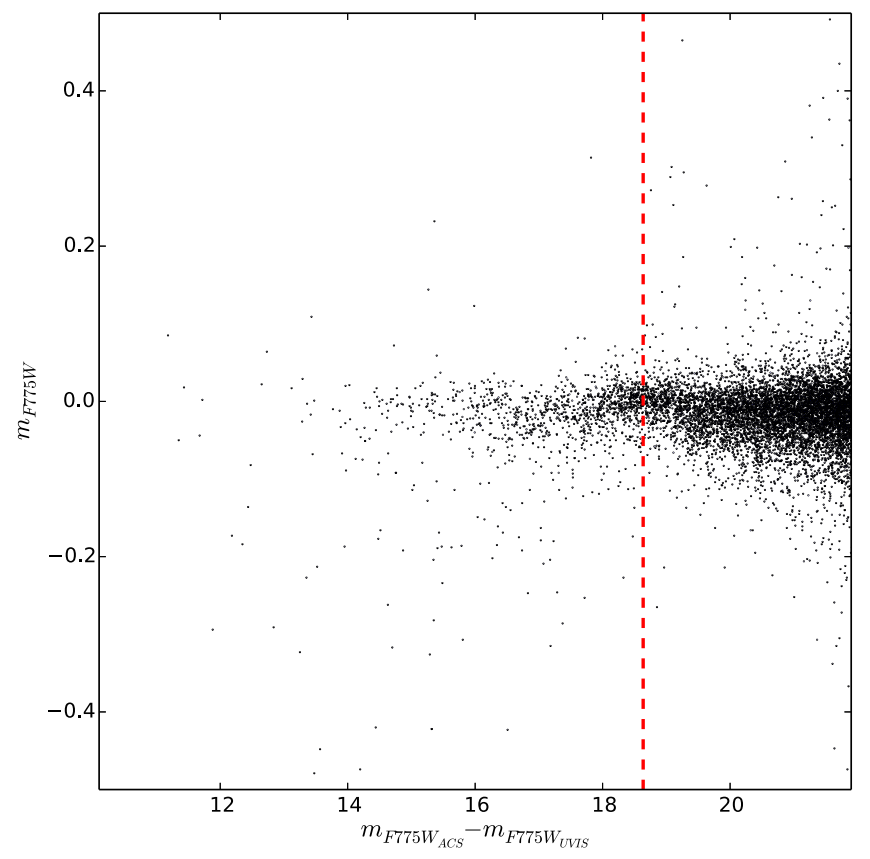

Figure 7. Magnitude difference for the stars measured in both the F775W filters as a function of the $m_{\mathrm{F} 775 \mathrm{~W}_{\mathrm{ACS}}}$ magnitude. The vertical red dashed line marks the saturation point for the $\mathrm{F} 775 \mathrm{~W}_{\mathrm{ACS}}$ data.

Stars too faint to be detected in one filter (negative flux) or that fall outside the filter field of view have been assigned a magnitude of 99.999. We assigned to each source in each filter a quality flag $f_{\text {filter }}$ ranging from 1 to 7 . Stars that cannot be detected in one filter because they are outside the filter coverage have been assigned $f_{\text {filter }}=7$, while stars that are too faint to be measured have $\mathrm{f}_{\text {filter }}=6$.

We tested several combinations of diagnostics to remove as many spurious detections as possible. At the end, we found that eliminating the sources with $Q_{\text {fit }}<0.75$ removes the majority of the outliers, giving the cleanest CMDs without severely compromising the completeness of the catalog. Figure 10 shows that a consequence of the selection in $Q_{\text {fit }}$ is the cut at the fainter magnitudes. As shown in Figure 9, these are also the sources with larger photometric errors, therefore it is not a surprise that these objects were not measured as well as those characterized by higher $Q_{\text {fit }}$ values. Sources that have been well fitted by our PSF model are likely stars. If their luminosities have been measured in more than one _flt/_flc image, they have been assigned $f_{\text {filter }}=1$.

The photometric errors (Figure 9) are determined using the formula $\sigma_{\mathrm{mag}}=1.1 \sigma_{\text {flux }} /$ flux, where $\sigma_{\text {flux }}$ is the standard deviation of the independent measurements. For the sources detected in just one exposure, it was not possible to measure a photometric error. We assigned to these stars the most probable error for their magnitude and marked them using the flag $\mathrm{f}_{\text {filter }}=2$ if they were characterized by a good quality of the PSF fitting $\left(Q_{\text {fit }}>0.75\right)$; otherwise we used $f_{\text {filter }}=5$. Sources with photometric errors smaller than 0.25 , but with a poor fit of the PSF, have been assigned flag $\mathrm{f}_{\text {filter }}=3$, while objects with photometric errors larger than 0.25 and poor quality of the PSF fitting have been flagged with $\mathrm{f}_{\text {filter }}=4$.

In total, we detected more than 820,000 sources. Of these sources, $\sim 620,000$ have been flagged with $\mathrm{f}_{\text {filter }}=1$ in at least one of the F775W filters $(\sim 19,000$ stars are in common between ACS and WFC3, 330,000 stars are in the area

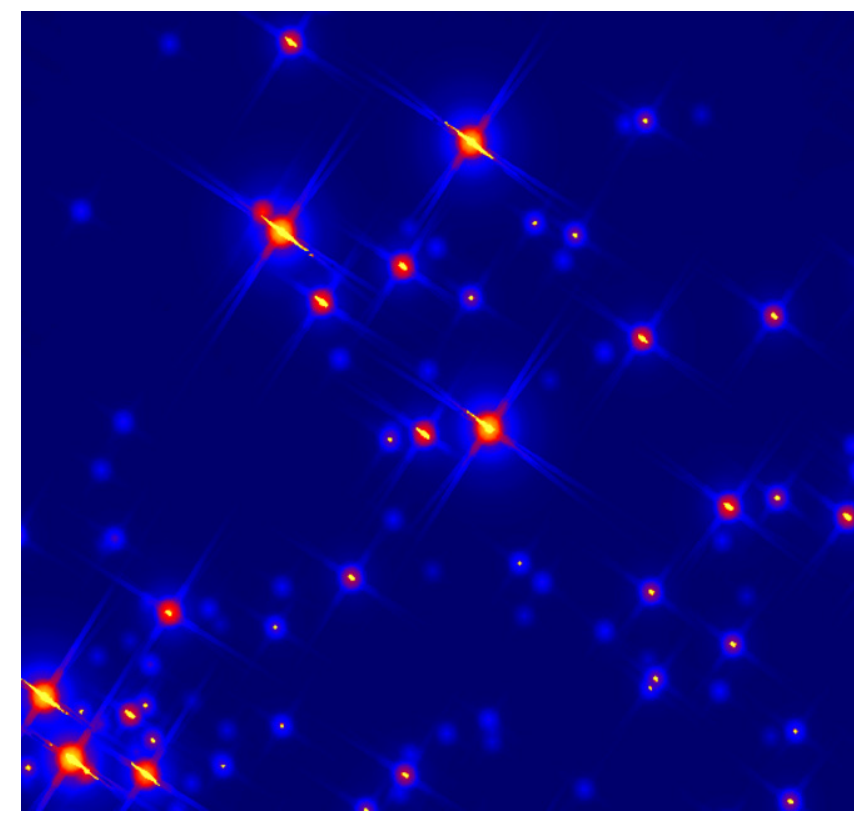

Figure 8. Detail of the photometry mask created by KS2 for the filter F555W. Because of the overlap of exposures acquired in different visits, with slightly different rotation angles, some stars show multiple diffraction spikes.

covered only with ACS, and 270,000 in WFC3 only). More than 520,000 stars have $\mathrm{f}_{\text {filter }}=1$ in the F110W filter and $\sim 570,000$ in F160W. Finally, there are more than 30,000 stars with $f_{\text {filter }}=1$ in the $\mathrm{F} 275 \mathrm{~W}$ filter, more than 100,000 in $\mathrm{F} 336 \mathrm{~W}, \sim 400,000$ in F555W, and $\sim 130,000$ in F658N. The absolute astrometry of the catalog was derived using 2MASS. J2000.0 R.A. and decl. in sexagesimal units were also used to define the source IDs in the catalog. An extract of the catalog is shown in Table 3. The table reports for each source listed the identification number ID, the magnitude $\mathrm{m}$, the photometric error err, the quality of the PSF-fitting parameter $Q_{\text {fit }}$, and the quality flag $f$ for the filters $\mathrm{F} 555 \mathrm{~W}$ and $\mathrm{F} 775 \mathrm{~W}_{\mathrm{ACS}}$. Coordinates in pixels $x$ and $y$ and celestial coordinates in degrees and Ra and Dec are also listed.

The final catalog is available for download from the Mikulsky Archive for Space Telescopes (MAST) at https:// doi.org/10.17909/T9RP4V. For each of the HTTP filter, we also created a larfge mosaic using astrodrizzle. ${ }^{22}$ Each mosaic can be downloaded from the url https://doi.org/10.17909/ T9RP4V as well.

\subsection{Artificial Star Tests}

Artificial star tests are a standard procedure to assess the level of completeness and accuracy of a photometric analysis. The tests are performed by inserting stars with known positions and fluxes into the data set, and then repeating the photometric analysis as was used for the real stars. The selection criteria applied to the observed catalog to discard spurious detections are applied to the recovered artificial stars as well.

The difference between the input and output magnitudes of the recovered artificial stars provides an estimate of the photometric error. The fraction of recovered artificial stars per bin of magnitude gives an estimate of the photometric completeness.

\footnotetext{
${ }^{22}$ http://drizzlepac.stsci.edu
} 


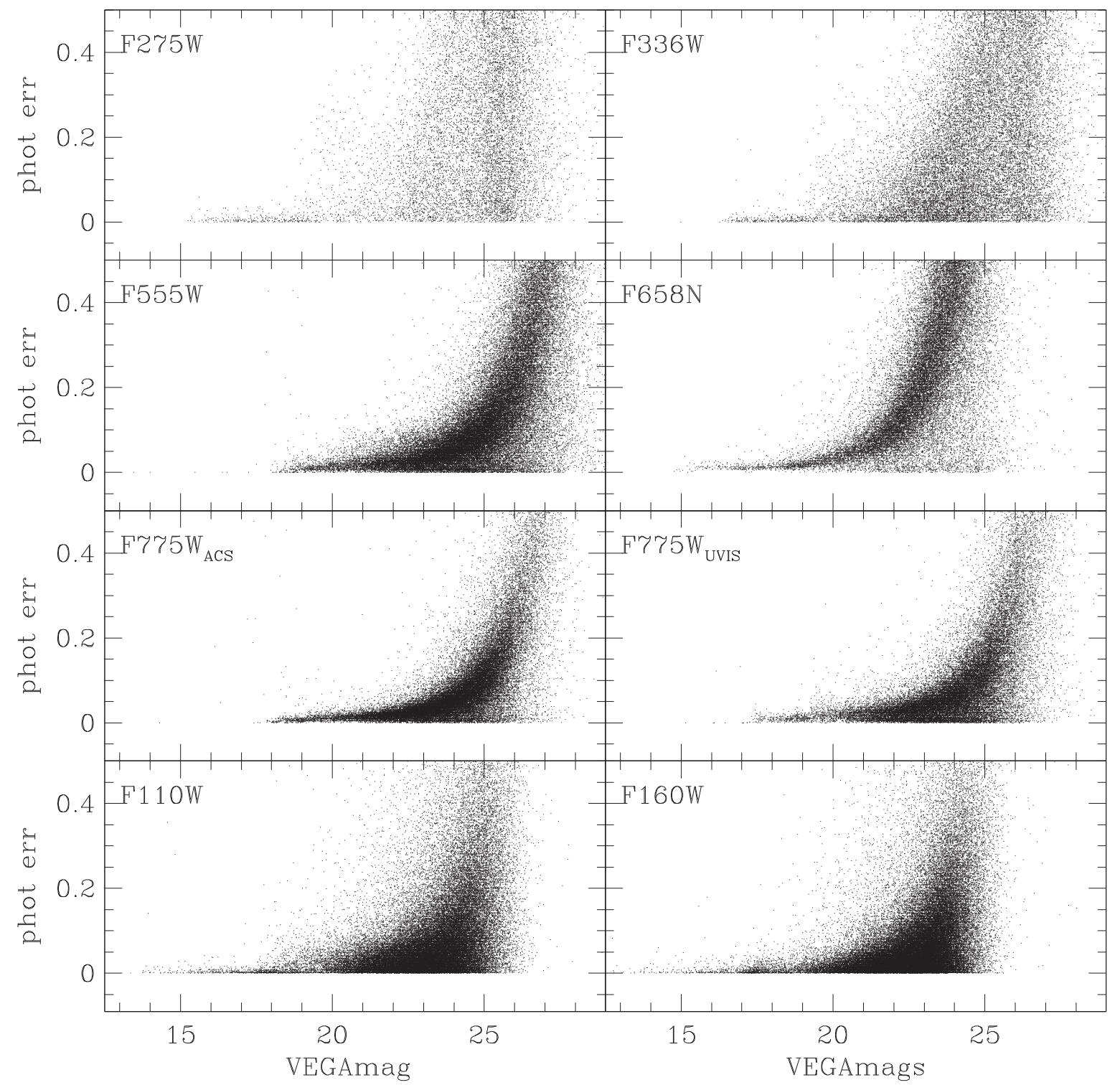

Figure 9. Standard deviation of the independent magnitude measurements. From top to bottom, left to right we show the filters F275W, F336W, F555W, F658N, ACS/F775W, UVIS/F775W, F110W, and F160W. In each plot only 5\% of the sources are shown.

In total, we simulated almost 20 million artificial stars in each filter. Input magnitudes of the artificial stars in the various filters have been set up to reproduce the same range of colors covered by the observed data. KS2 allows us to add artificial stars on a grid. To avoid the concern that artificial stars may interact with each other, we imposed a 20 pixel minimum distance between one artificial star and the nearest simulated source.

As in the case of the photometry, all the filters were analyzed at the same time. The input coordinates of the artificial stars were defined in the reference frame, and then KS2 simulated each artificial star on all the single _flt/_flc images corresponding to that position.

Once the artificial stars were added to the various frames, we repeated the photometry as described in Section 3. In our analysis, we considered an artificial star as recovered in a filter if its input and output fluxes agreed to within $0.75 \mathrm{mag}$. Larger magnitude differences in fact would imply that the flux of the detected object is dominated by a brighter real source. As in the real stars photometric analysis, we also required that each star is found with $Q_{\text {fit }}>0.75$.

Figure 11 shows the completeness levels for different regions across the Tarantula Nebula for each of the HTTP filters. As expected, crowding is the main source of incompleteness, as shown by the fact that around NGC 2070 even the deepest exposures (F55WW, F775W $\mathrm{W}_{\mathrm{ACS}}$, and

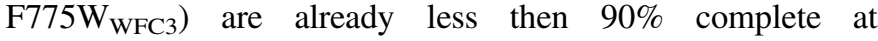
mag $=22$, while in less crowded regions (i.e., NGC 2060 and in the field) in these filters the completeness remains above $90 \%$ down to mag $=24.5-25$.

Not surprisingly, the photometry in the narrow band filter F658N is less complete in all regions, dropping below $90 \%$ between 19 and 21 depending on the crowding. It is interesting to note that the photometry in the NUV (F275W and F336W) remains almost $100 \%$ down to mag $=23$, mimicking very well the completeness level of the broadband optical filters. However, while for the latter the completeness decays more smoothly, in the NUV it rapidly drops to zero. This can 


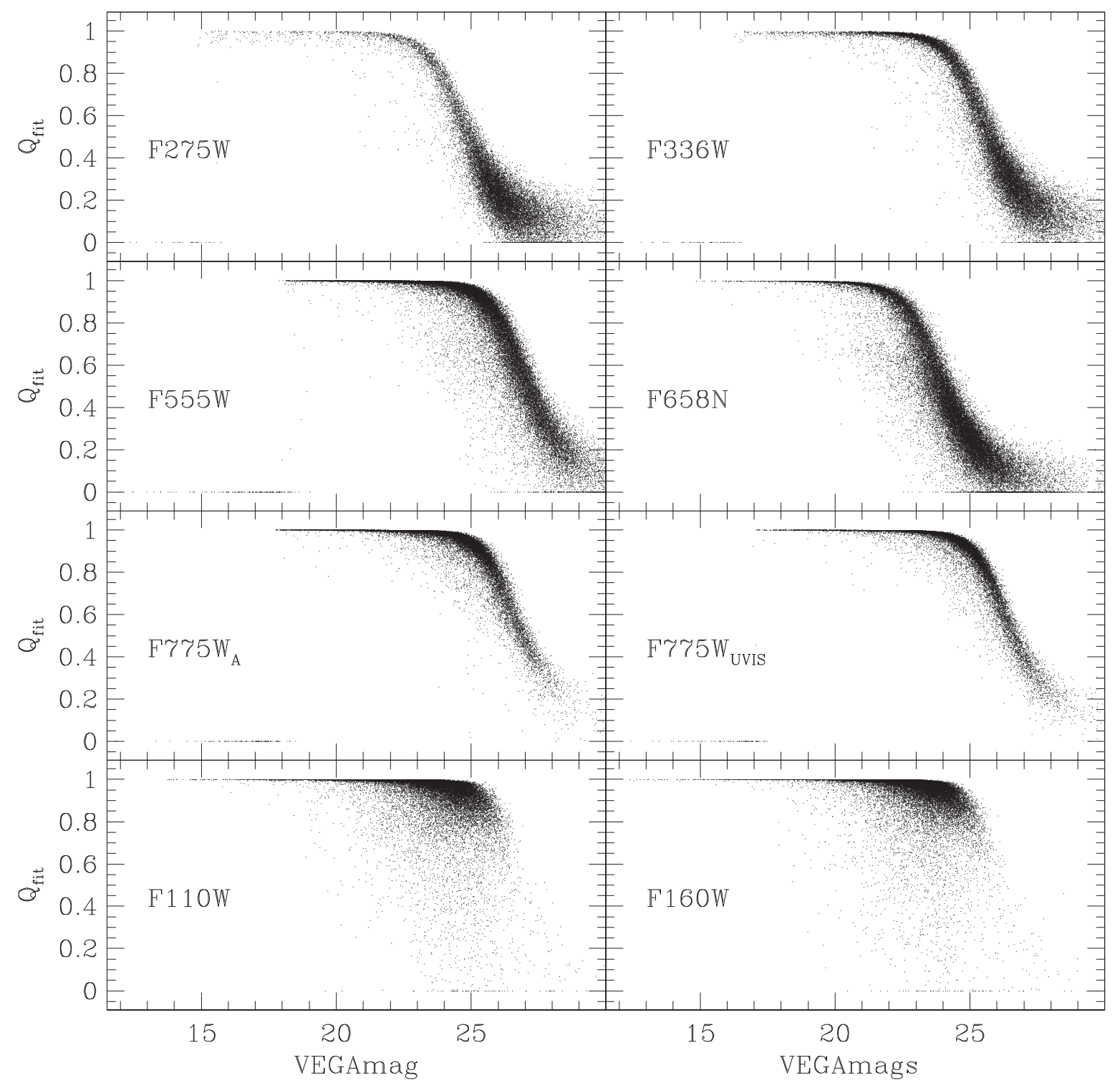

Figure 10. Quality of the PSF fitting as a function of the apparent magnitude for the filters F275W, F336W, F555W, F658N, ACS/F775W, UVIS/F775W, F110W, and F160W. In each plot only 5\% of the sources are shown.

probably be ascribed to the fact that in the NUV we forced the detection.

Finally, the completeness declines in the NIR is more gentle than in in the broadband optical filters. As a result, although even in regions of moderate crowding the NIR photometry is already less then $90 \%$ complete at mag $=22.5$, its detection threshold is as deep as in the optical.

\section{COLOR-MAGNITUDE DIAGRAMS}

CMDs realized from the HTTP photometric catalog can be used to interpret the stellar populations found in 30 Doradus. CMDs at near-ultraviolet (NUV; $m_{\mathrm{F} 275 \mathrm{~W}}$ versus $m_{\mathrm{F} 275 \mathrm{~W}}-$ $m_{\mathrm{F} 336 \mathrm{~W}}$, panel (a)), optical $\left(m_{\mathrm{F} 336 \mathrm{~W}}\right.$ versus $m_{\mathrm{F} 336 \mathrm{~W}}-m_{\mathrm{F} 555 \mathrm{~W}}$, and $m_{\mathrm{F} 555 \mathrm{~W}}$ versus $m_{\mathrm{F} 555 \mathrm{~W}}-m_{\mathrm{F} 775 \mathrm{~W}}$, panels (b) and (c) respectively), and near-infrared (NIR; $m_{\mathrm{F} 110 \mathrm{~W}}$ versus $m_{\mathrm{F} 110 \mathrm{~W}}-m_{\mathrm{F} 160 \mathrm{~W}}$, panel (d)) wavelengths are shown in Figure 12. In plotting the optical CMD, we combined the ACS and UVIS F775W magnitudes, and when both magnitudes were available we used the average value. Evolutionary phases for stars of different ages and masses are highlighted in each CMD to aid with the interpretation.

An inspection of the four CMDs shows that a variety of stellar populations are present in the region of the Tarantula Nebula. Because of the wide wavelength coverage, the shown CMDs can be used to highlight the properties of stars of different ages and in different evolutionary phases.

\subsection{The NUV CMD}

The most prominent feature in the NUV CMD (Figure 12panel (a)) is the narrow and well defined upper main sequence (UMS) characterized by stars brighter than $m_{\mathrm{F} 275 \mathrm{~W}} \leqslant 19.5$, and bluer than $m_{\mathrm{F} 275 \mathrm{~W}}-m_{\mathrm{F} 336 \mathrm{~W}} \leqslant 0.25$. The UMS is typical of a young stellar population and comprises intermediate$\left(M \gtrsim 4 M_{\odot}\right)$ and high-mass stars.

Figure 13 shows the NUV CMD with superimposed Padova isochrones (Bressan et al. 2012; Chen et al. 2014; Tang et al. 2014) for different ages. We assumed the distance modulus of 18.5, consistent with the literature (i.e., Panagia 
Table 3

HTTP Photometric Catalog

\begin{tabular}{|c|c|c|c|c|c|c|c|c|c|c|c|c|c|c|}
\hline ID & & $m$ & $\begin{array}{l}\text { F55 } \\
\text { err }\end{array}$ & $Q$ & $f$ & $m$ & $\begin{array}{l}\text { F775V } \\
\text { err }\end{array}$ & $Q$ & $f$ & & $\begin{array}{c}X \\
\text { (pixels) }\end{array}$ & $\begin{array}{c}Y \\
\text { (pixels) }\end{array}$ & $\begin{array}{c}\text { R.A. } \\
\text { (degree) }\end{array}$ & $\begin{array}{c}\text { decl. } \\
\text { (degree) }\end{array}$ \\
\hline SABBI 053850.819-691404.92 & $\ldots$ & 24.528 & 0.349 & 0.444 & 1 & 24.163 & 0.016 & 0.992 & 1 & $\ldots$ & 17895.980 & 3636.450 & 84.711746 & -69.234700 \\
\hline SABBI 053851.156-691400.99 & $\ldots$ & 24.896 & 0.921 & 0.373 & 1 & 25.331 & 0.139 & 0.930 & 1 & $\ldots$ & 17801.800 & 3691.600 & 84.713150 & -69.233608 \\
\hline SABBI 053850.777-691403.44 & $\ldots$ & 19.384 & 0.018 & 0.989 & 1 & 19.225 & 0.005 & 1.000 & 1 & $\ldots$ & 17878.980 & 3670.330 & 84.711571 & -69.234289 \\
\hline SABBI 053850.977-691405.11 & $\ldots$ & 23.503 & 0.342 & 0.758 & 1 & 23.523 & 0.014 & 0.997 & 1 & $\ldots$ & 17881.240 & 3620.410 & 84.712404 & -69.234753 \\
\hline SABBI 053850.848-691404.25 & $\ldots$ & 24.860 & 0.648 & 0.309 & 1 & 25.089 & 0.004 & 0.952 & 1 & $\ldots$ & 17883.060 & 3648.090 & 84.711867 & -69.234514 \\
\hline SABBI 053851.098-691400.90 & $\ldots$ & 25.496 & 1.185 & 0.205 & 1 & 25.108 & 0.120 & 0.942 & 1 & $\ldots$ & 17806.840 & 3697.850 & 84.712908 & -69.233583 \\
\hline SABBI 053850.553-691403.61 & $\ldots$ & 22.614 & 0.184 & 0.913 & 1 & 22.579 & 0.041 & 0.999 & 1 & $\ldots$ & 17906.060 & 3684.190 & 84.710638 & -69.234336 \\
\hline SABBI 053850.382-691402.62 & $\ldots$ & 24.774 & 0.359 & 0.434 & 1 & 24.473 & 0.026 & 0.991 & 1 & $\ldots$ & 17910.450 & 3717.820 & 84.709925 & -69.234061 \\
\hline SABBI 053850.525-691404.28 & $\ldots$ & 25.702 & 0.401 & 0.071 & 1 & 25.169 & 0.121 & 0.958 & 1 & $\ldots$ & 17918.860 & 3672.460 & 84.710521 & -69.234522 \\
\hline SABBI 053850.700-691406.15 & $\ldots$ & 21.985 & 0.071 & 0.967 & 1 & 21.540 & 0.031 & 0.999 & 1 & $\ldots$ & 17926.770 & 3620.400 & 84.711250 & -69.235042 \\
\hline
\end{tabular}



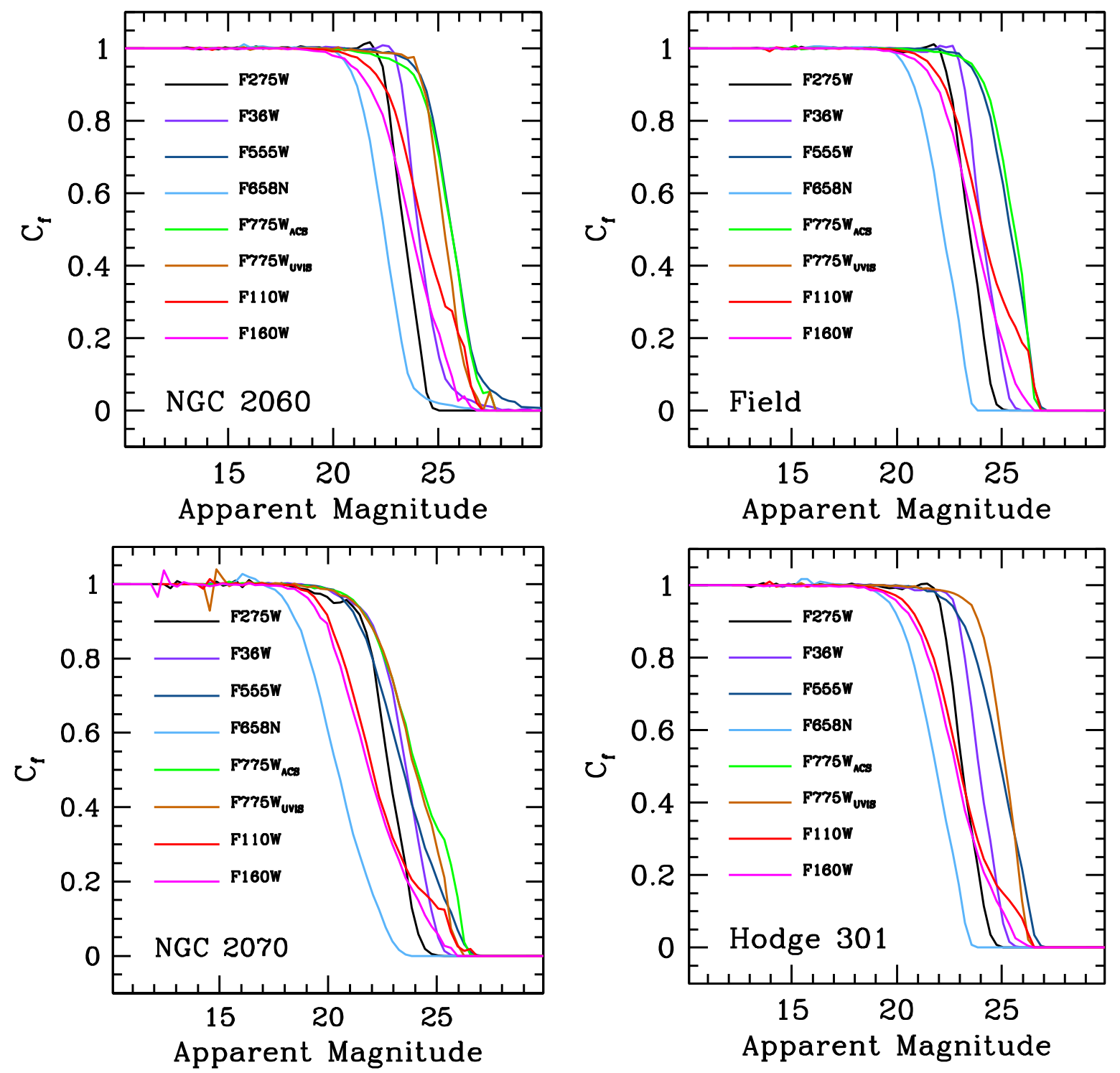

Figure 11. Ratio between the numbers of recovered and simulated artificial stars as a function of magnitude for all the HTTP filters around NGC 2070 (lower left panel), NGC 2060 (upper left panel), the field (upper right panel), and Hodge 301 (lower left panel). Different filters have been plotted using different colors.

et al. 1991; Pietrzyński et al. 2013), and the extinction law of de Marchi et al. (2016). For the younger stellar populations, we assumed a metallicity $Z=0.008$, (as proposed by Dufour et al. 1982; Bernard et al. 2008). The comparison with theoretical models indicates that the brightest stars in the CMD are likely O-type dwarfs as massive as $\sim 60-80 M_{\odot}$.

At magnitude $m_{\mathrm{F} 275 \mathrm{~W}} \simeq 19.4$, the UMS bends toward redder colors (see the inset in Figure 13). This is likely where young intermediate-mass pre-main sequence (PMS) stars join the UMS. This point is often called PMS turn-on (TOn). The brighter TOn can be reproduced with a $1 \mathrm{Myr}$ old isochrone and $E(B-V)=0.3$. Fainter TOns can be found down to $\sim 5-10 \mathrm{Myr}$, suggesting that the region has been forming stars for a prolonged time, in agreement with the fact that the stellar population of the 30 Doradus region covers multiple ages, from regions of ongoing star formation (Rubio et al. 1992, 1999; Walborn et al. 1999; Brandner et al. 2001; Walborn. et al. 2013 ) to the 15-25 Myr old Hodge 301 (Grebel \& Chu 2000; Evans et al. 2015).
The majority of the brighter and younger PMS stars are within $10-15 \mathrm{pc}$ from the center of R136 (R.A. $J 2000=$ $05^{\mathrm{h}} 38^{\mathrm{m}} 42^{\mathrm{s}} 3$ decl. $_{J 2000}=-69^{\circ} 06^{\prime} 03{ }^{\prime \prime} \cdot 3$ Hog et al. 2000), consistent with the fact that about one-fourth of the $\mathrm{O}$ stars of the LMC are found in NGC 2070 (Kennicutt et al. 1989). The NUV CMD for the NGC 2070 region is shown in Figure 14. Unfortunately the southern part of NGC 2070 falls within the gap that affects the mosaic in the F275W filter (Figure 3); therefore, this CMD underestimates the total number of UV sources in this region.

While Padova ischrones reproduce very well the properties of the UMS and of the RC, therefore supporting the validity of the assumed extinction law, they fail in reproducing the colors of the PMS candidates. PMSs in particular are constantly bluer than what is predicted by the models. This discrepancy is likely due to problems with the models of atmosphere, and in particular to the characteristic excess radiation shortward of the Balmer discontinuity due to accretion (Kuhi 1974; Gullbring et al. 1998; Robberto et al. 2004). 


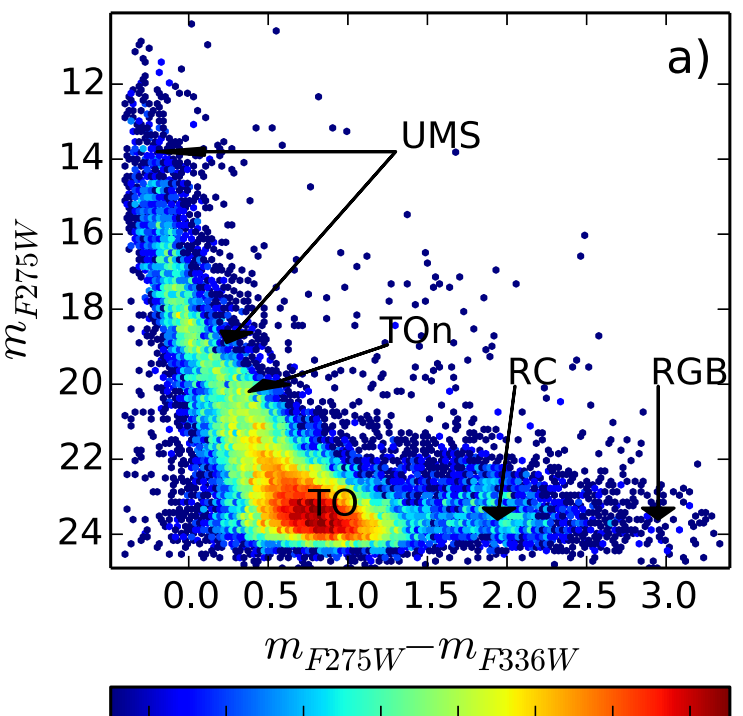

$\begin{array}{llllllll}0.4 & 0.6 & 0.8 & 1.0 & 1.2 & 1.4 & 1.6 & 1.8\end{array}$ $\log ($ counts $)$

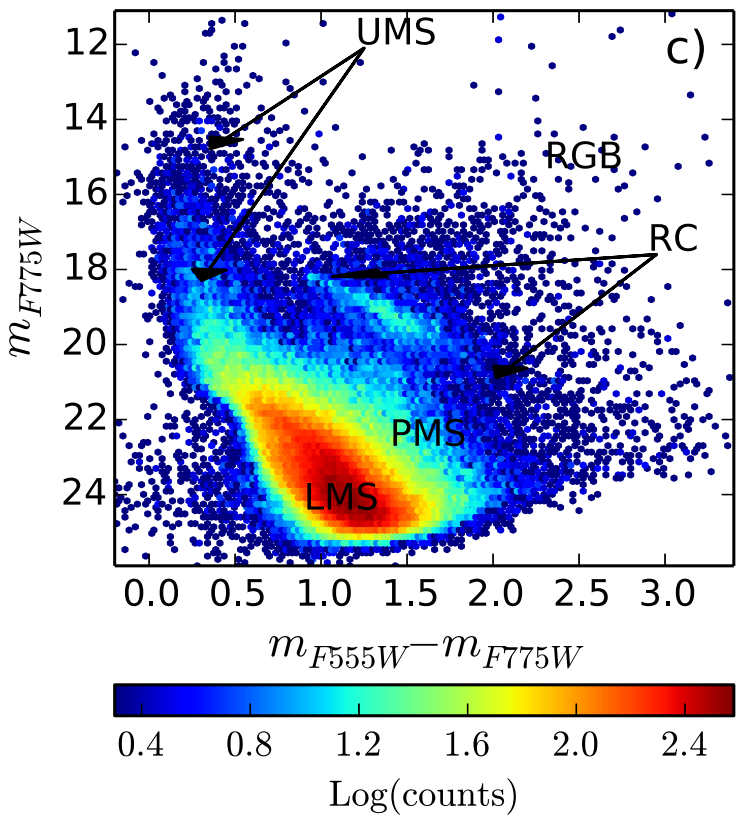

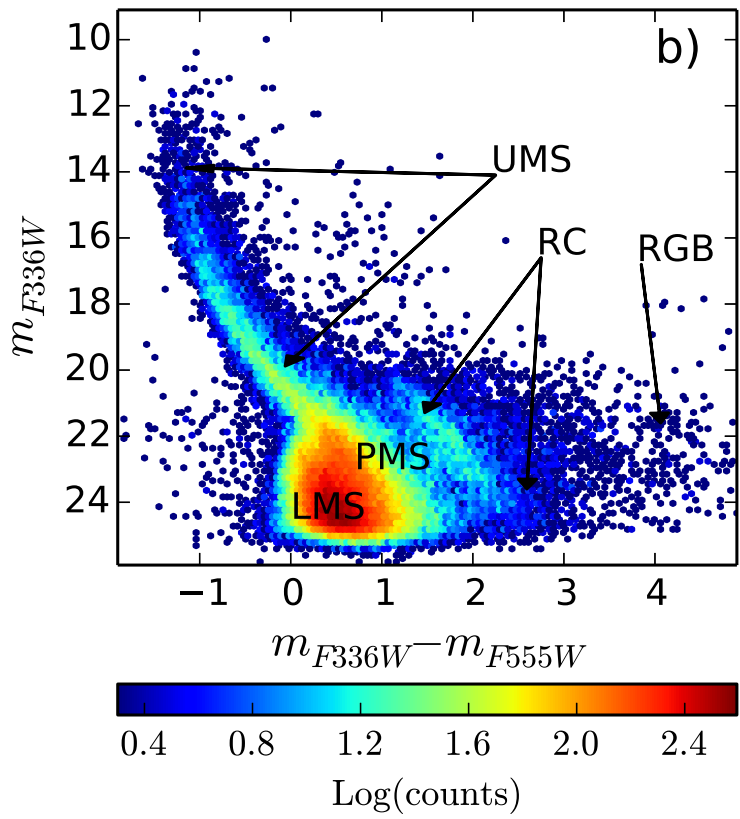

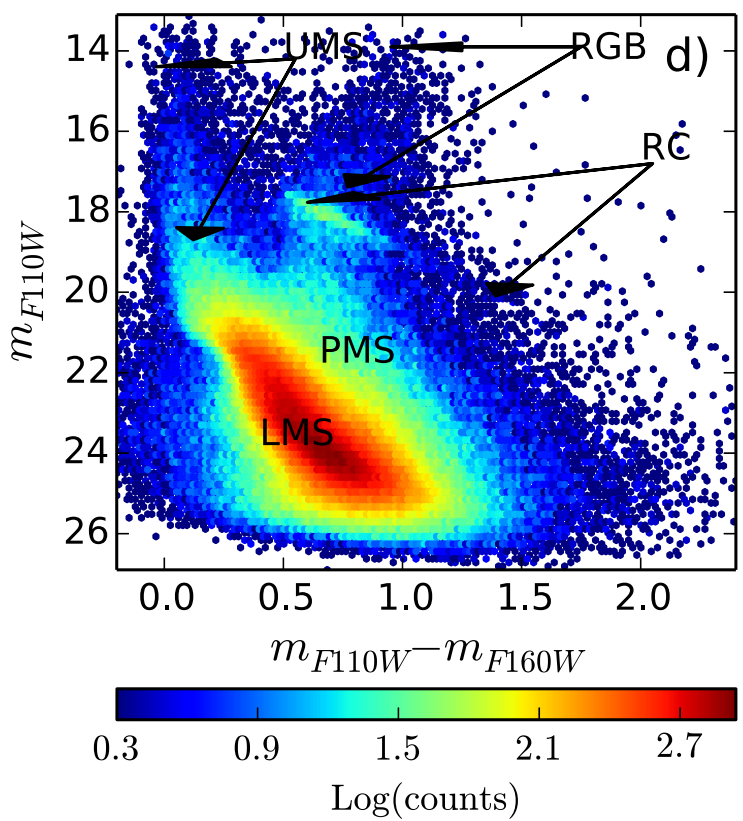

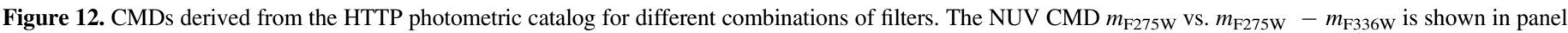

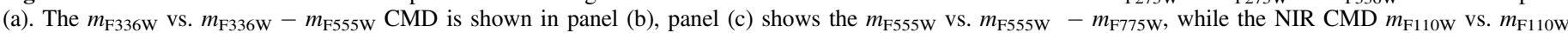
$-m_{\mathrm{F} 160 \mathrm{~W}}$ is shown in panel (d). The position of evolutionary phases typical of stars of different ages and masses are indicated in all four CMDs.

Moving away from NGC 2070, the contamination from older main sequence and turn-off (TO) stars may become important. For example, the TO of a $\sim 1$ Gyr old stellar population affected only by the Galactic foreground extinction would be as bright as $m_{\mathrm{F} 275 \mathrm{~W}} \simeq 20$. To better understand the origin of the bending in the UMS observed in Figure 13), we compared the spatial distribution of the stars found at luminosity of the TOn with that of fainter and bluer sources, which likely belong to the older lower main sequence (LMS), and to the red clump stars (RC; corresponding to a stellar population older than 1-2 Gyr.).

We used a Kolmogorov-Smirnov test to estimate the probability that TOn candidates have the same spatial distribution of RC or LMS stars (Figure 15). In our analysis, we divided the TOns candidates in two groups. The bright TOn
(bTOn) stars are in the magnitude range $19.5<m_{\mathrm{F} 275 \mathrm{~W}}<20$, and are bluer than $m_{\mathrm{F} 275 \mathrm{~W}}-m_{\mathrm{F} 336 \mathrm{~W}} \leqslant 0.5$. The faint $\mathrm{TOn}$ (fTOn) are in the magnitude range $21<m_{\mathrm{F} 275 \mathrm{~W}}<21.5$ and are bluer than $m_{\mathrm{F} 275 \mathrm{~W}}-m_{\mathrm{F} 336 \mathrm{~W}} \leqslant 0.5$. We consider LMS stars those sources fainter than $m_{\mathrm{F} 275 \mathrm{~W}} \geqslant 22$ and bluer than $m_{\mathrm{F} 275 \mathrm{~W}}-m_{\mathrm{F} 336 \mathrm{~W}} \leqslant 1.5$, while sources redder than $m_{\mathrm{F} 275 \mathrm{~W}}-m_{\mathrm{F} 336 \mathrm{~W}}>1.5$ are considered $\mathrm{RC}$ stars.

While we cannot reject the hypothesis that bTOn and fTOn candidates, or LMS and RC stars, are extracted from the same distribution, we can exclude that TOns candidates are compatible with LMS (the probability is $p \leqslant 10^{-8}$ for bTOn and $p \leqslant 10^{-12}$ for fTOn candidates) or RC stars ( $p \leqslant 10^{-6}$ for bTOn and $p \leqslant 10^{-7}$ for fTOn candidates), supporting the hypothesis that the change in the slope of the UMS at $m_{\mathrm{F} 275 \mathrm{~W}}=19.5$ is mainly due to the MS-TOn. 


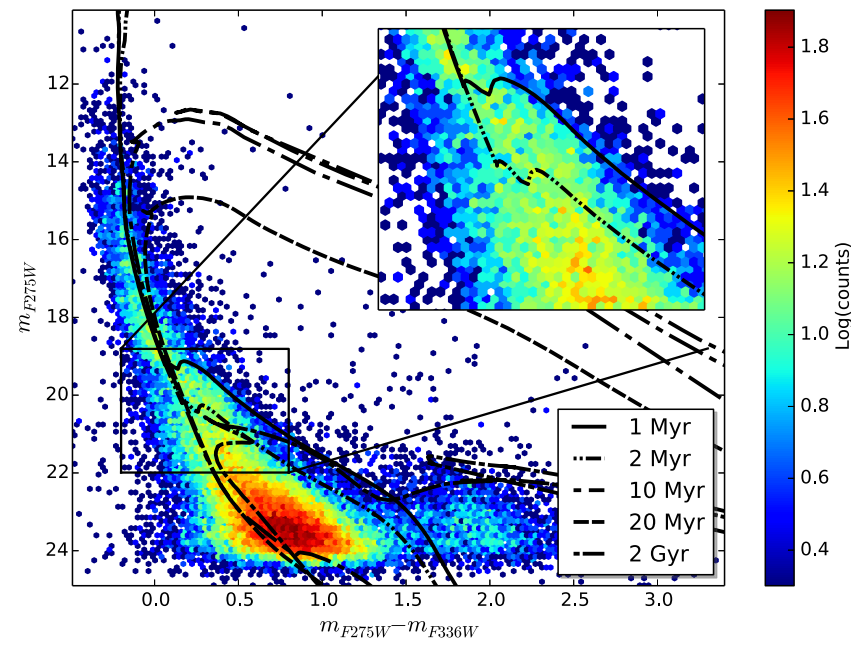

Figure 13. NUV CMD $m_{\mathrm{F} 275 \mathrm{~W}}$ vs. $m_{\mathrm{F} 275 \mathrm{~W}}-m_{\mathrm{F} 336 \mathrm{~W}} \mathrm{CMD}$ for the entire region covered by HTTP. Padova isochrones for metallicity for 1 (dasheddotted-dotted line), 2 (long-short dashed line), 5 (dotted line) Myr, and age $=2.0 \mathrm{Gyr}$ (continuous line) are superimposed assuming a distance modulus of 18.5 , and a reddening $E(B-V)=0.3$ in addition to the Galactic foreground extinction. Extinction coefficients $A_{\mathrm{F} 275 \mathrm{~W}}=7.9$ and $A_{\mathrm{F} 336 \mathrm{~W}}=$ 6.4 are from De Marci et al. (2015). For the younger populations (age $\leqslant$ $5 \mathrm{Myr}$ ), we assumed metallicity $Z=0.008$, while for the older populations we assumed $Z=0.004$. The locus of the PMS-TOn is shown in the zoomed in inset.

\subsection{Optical and NIR CMDs}

The optical and NIR CMDs (Figure 12-panels (c) and (d)) can be used to study the properties of the stellar populations that formed in the region during the LMC's lifetime. The older stars in the CMDs belong to the LMC field. Evolutionary sequences typical of these older stellar populations are the LMS, the subgiant branch (SGB), the red giant branch (RGB), and the RC.

The LMS are low-mass $\left(<2 M_{\odot}\right)$ stars with ages spanning from a few tens of million of years to several billion years. In the optical (panel (c)) of Figure 12), most of LMS stars are fainter than $m_{\mathrm{F} 775 \mathrm{~W}} \geqslant 19$ and bluer than $m_{\mathrm{F} 555 \mathrm{~W}}-m_{\mathrm{F} 775 \mathrm{~W}}<$ $0.17 m_{\mathrm{F} 775 \mathrm{~W}}-2.6$, while in the NIR (panel (d)) are fainter than $m_{\mathrm{F} 110 \mathrm{~W}} \geqslant 18$ and bluer than $m_{\mathrm{F} 110 \mathrm{~W}}-m_{\mathrm{F} 160 \mathrm{~W}}<$ $0.16 m_{\mathrm{F} 110 \mathrm{~W}}-3$.

Once stars evolve off the main sequence, they become brighter and redder $\left(m_{\mathrm{F} 775 \mathrm{~W}}<21.5, m_{\mathrm{F} 555 \mathrm{~W}}-m_{\mathrm{F} 775 \mathrm{~W}}>1\right.$ in panel (c), and $m_{\mathrm{F} 110 \mathrm{~W}}<20.5, m_{\mathrm{F} 110 \mathrm{~W}}-m_{\mathrm{F} 160 \mathrm{~W}}>0.7$ in panel (d), respectively) and migrate into the SGB and then RGB evolutionary phases. Padova isochrones for different ages and metallicities have been superimposed on the optical CMD in Figure 16 and on the NIR CMD in Figure 17 to help with the interpretation.

As in the NUV, in both optical and NIR CMDs the brighter and bluer objects are the UMS stars. In the optical CMD, they are brighter than $m_{\mathrm{F} 775 \mathrm{~W}}<19$ and bluer than $m_{\mathrm{F} 555 \mathrm{~W}}$ $-m_{\mathrm{F} 775 \mathrm{~W}}<1$, while in the NIR they occupy the portion of CMD above $m_{\mathrm{F} 110 \mathrm{~W}}<18$, with colors bluer than $m_{\mathrm{F} 110 \mathrm{~W}}$ $-m_{\mathrm{F} 160 \mathrm{~W}}<0.4$.

The majority of the faint $\left(m_{\mathrm{F} 775 \mathrm{~W}}<19\right)$ red $\left(m_{\mathrm{F} 555 \mathrm{~W}}\right.$ $\left.-m_{\mathrm{F} 775 \mathrm{~W}}>0.17 m_{\mathrm{F} 775 \mathrm{~W}}-2.6\right)$ objects in panel (c) of Figure 12 (corresponding in panel (d) to the stars fainter than $m_{\mathrm{F} 110 \mathrm{~W}}<18$, and redder than $m_{\mathrm{F} 110 \mathrm{~W}}-m_{\mathrm{F} 160 \mathrm{~W}}>$ $0.16 m_{\mathrm{F} 110 \mathrm{w}}-3$ ) are likely low-mass PMS stars. When looking at the CMDs for the entire region, a combination of differential

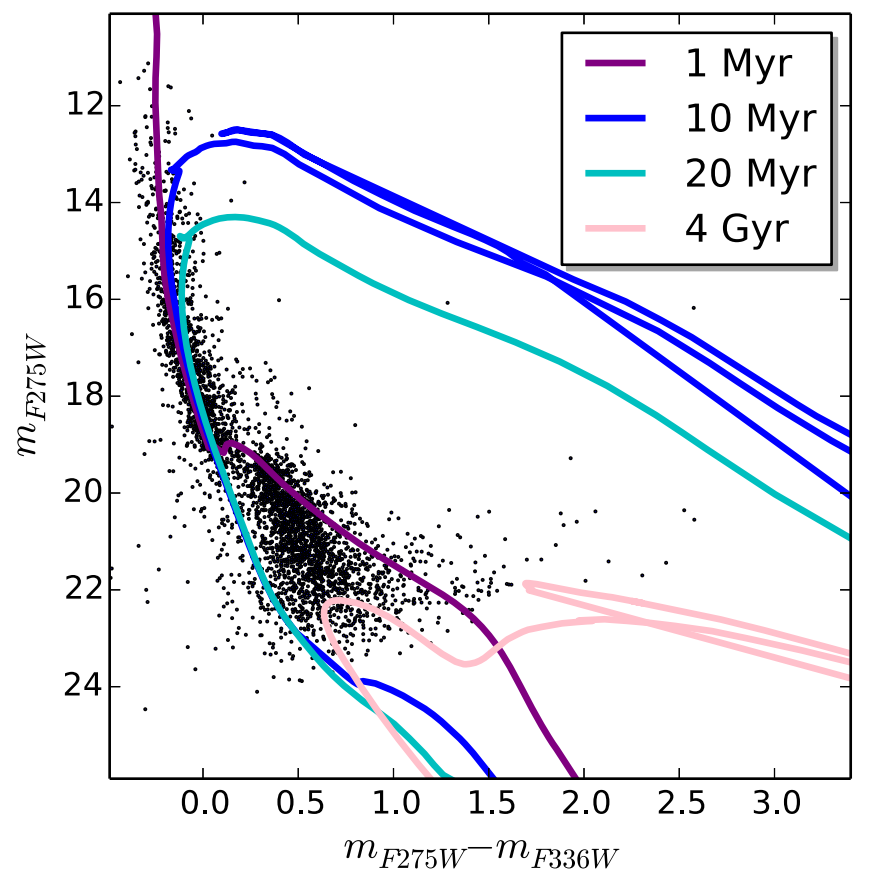

Figure 14. NUV CMD $m_{\mathrm{F} 275 \mathrm{~W}}$ vs. $m_{\mathrm{F} 275 \mathrm{~W}}-m_{\mathrm{F} 336 \mathrm{~W}} \mathrm{CMD}$ for the stars within $15 \mathrm{pc}$ from the center of R136. Padova isochrones for metallicity $Z=0.008$ and ages $=1,10$, and $20 \mathrm{Myr}$ are shown in purple, blue, and cyan, respectively. The assumed distance modulus is 18.5 and we added $E$ $(B-V)=0.28$ to the Galactic foreground reddening. A $4 \mathrm{Gyr}$ old isochrone for metallicity $Z=0.004$ is shown in pink assuming a distance modulus of 18.5 .

reddening (which pushes LMS stars toward redder colors) and age spread (older PMS stars are cooler and lie closer to the LMS than younger objects) blurs the transition between LMS and PMS starts. Rotational variability, accretion excess, unresolved binaries, and/or dusty disks (see, e.g., Gouliermis 2012) can further broaden the portion of the CMD populated by PMS stars, making it even harder to separate PMS stars from the LMS.

\subsection{Reddening and Dust Distribution}

RC stars are evolved core helium burning sources whose intrinsic luminosity is relatively insensitive to age. Once their metallicity is known, RC stars can be used as standard candles to determine distances (e.g., Stanek et al. 1998; Girardi \& Salaris 2001) and reddening (Udalski et al. 1999a, 1999b; Zaritsky et al. 2004; Haschke et al. 2011; De Marchi et al. 2014).

Figure 18 shows a detail of the entire optical CMD centered on the RC. RC stars are distributed along a narrow and extended strip whose stretch in magnitude and color indicates that the reddening is varying considerably across the region. While the Galactic extinction $\left(R_{V}=3.1\right.$ and $\left.A_{V}=0.06\right)$ is sufficient to fit the bluer edge of the RC, reddening values larger than $E(B-V)>1.0$ are necessary to reproduce the color of the most extinguished stars.

Figure 19 shows the spatial distribution of the RC stars that in Figure 18 fall within the blue rectangle. The stars have been superimposed on the mosaic in the F555W filter to help with the identification of main structures. Stars have been colorcoded from dark blue to red as a function of their color excess in the $m_{\mathrm{F} 555 \mathrm{~W}}-m_{\mathrm{F} 775 \mathrm{~W}}$ color, with the color of the $\mathrm{RC}$ 


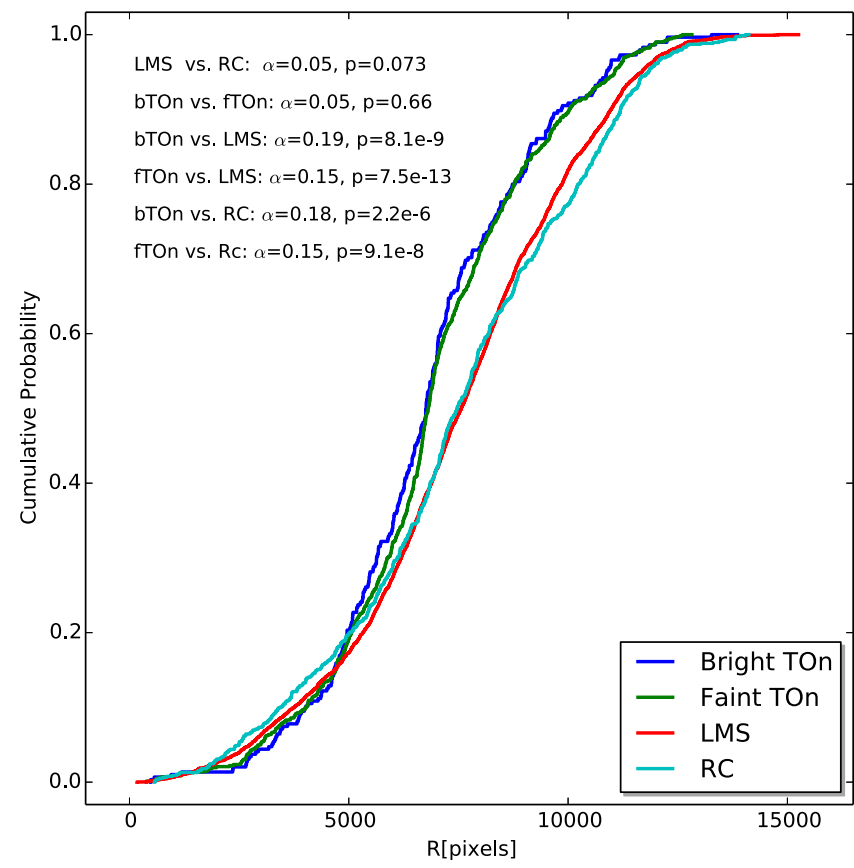

Figure 15. Cumulative radial distributions for the bright (blue line) and the faint (green line) TOn candidates, the LMS (red line), and the RC (cyan line) stars as a function of their projected distance $(R)$ from the center of the mosaic (R.A. $=05: 37: 52.285 ;$ decl. $=-69: 07: 21.57 ; J=2000)$. Results from KS tests comparing the spatial distribution of TOn candidates, LMS, and RCs are listed.

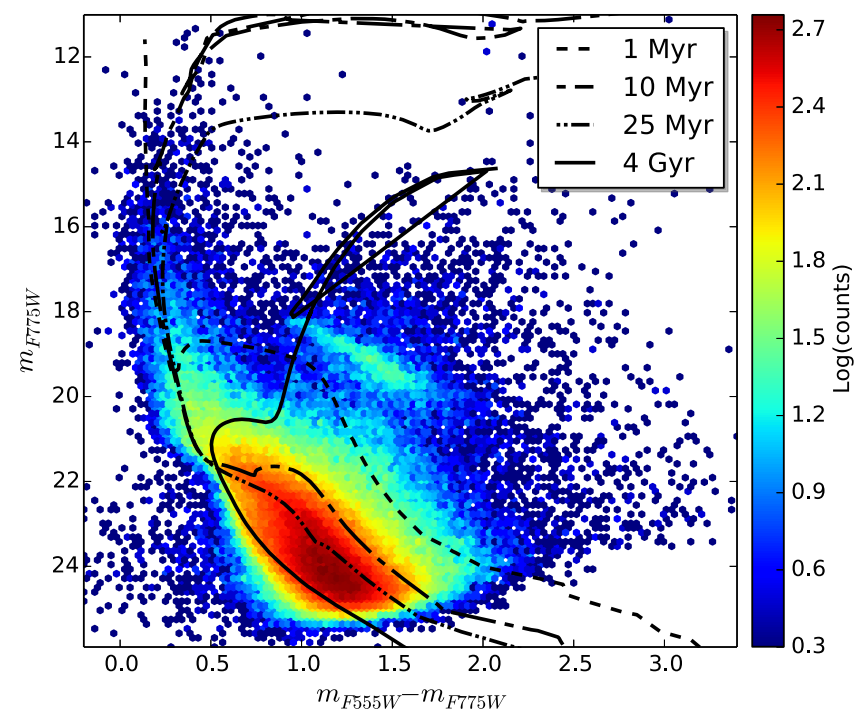

Figure 16. Optical $m_{\mathrm{F} 775 \mathrm{~W}}$ vs. $m_{\mathrm{F} 555 \mathrm{~W}}-m_{\mathrm{F} 775 \mathrm{~W}} \mathrm{CMD}$ for the entire region covered by HTTP. Padova isochrones for metallicity $Z=0.008$ and ages $=1$, 10, and $25 \mathrm{Myr}$ are superimposed using black dashed, short-dashed-longdashed, and dashed-dotted-dotted lines respectively. The continuous black line corresponds to a $4 \mathrm{Gyr}$ old isochrone with metallicity $Z=0.004$. For all isochrones, we assumed a distance modulus of 18.5. To fit the bluer edge of the younger ( $\leqslant 500 \mathrm{Myr}$ old) stellar populations, in addition to the Galactic extinction $\left(R_{V}=3.1\right.$ and $\left.A_{V}=0.06\right)$, a minimum $E(B-V)=0.3$ was needed. Extinction coefficients $A_{\mathrm{F} 555 \mathrm{~W}}=4.6$ and $A_{\mathrm{F} 775 \mathrm{~W}}=3.1$ are from De Marci et al. (2015). The Galactic foreground extinction was sufficient to fit the bluer edge of the RC.

affected only by the Galactic extinction being the zero point of the distribution.

Although the spectral energy distribution of few RC stars indicate that they are affected only by foreground

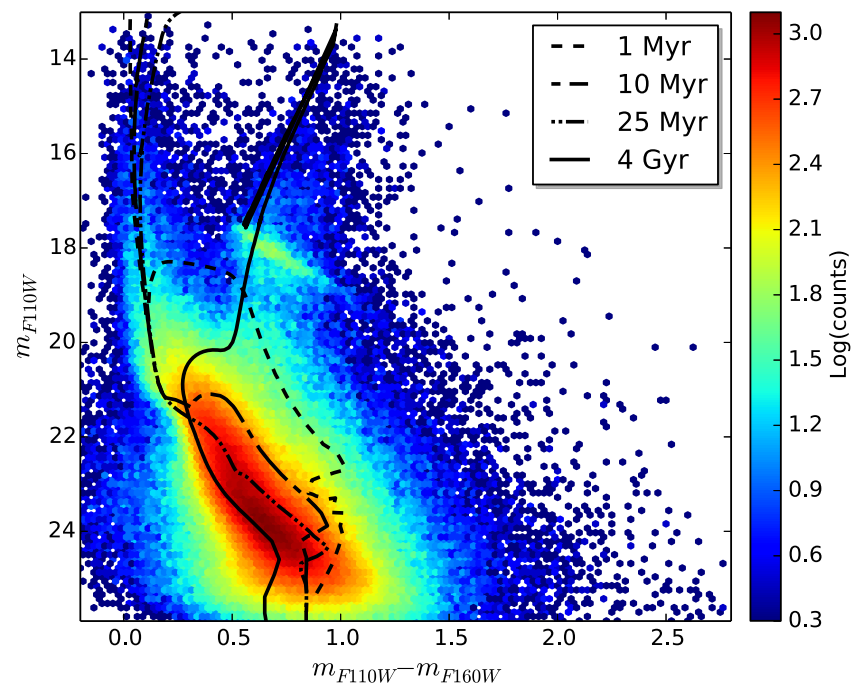

Figure 17. Same as Figure 16, but for the CMD in the NIR $m_{\mathrm{F} 110 \mathrm{w}}$ vs. $m_{\mathrm{F} 110 \mathrm{~W}}$ $-m_{\mathrm{F} 160 \mathrm{~W}}$. Extinction coefficients $A_{\mathrm{F} 110 \mathrm{~W}}=1.9$ and $A_{\mathrm{F} 160 \mathrm{~W}}=1.3$ are from De Marci et al. (2015).

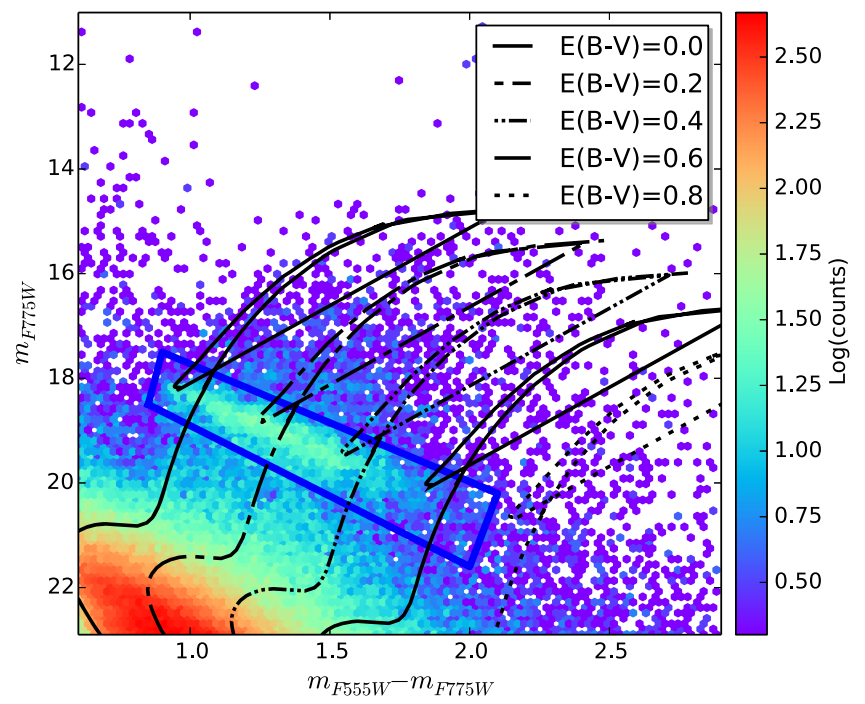

Figure 18. Detail of the $m_{\mathrm{F} 775 \mathrm{~W}}$ vs. $m_{\mathrm{F} 555 \mathrm{~W}}-m_{\mathrm{F} 775 \mathrm{~W}} \mathrm{CMD}$ centered around the RC. Padova isochrones for a $4 \mathrm{Gyr}$ old stellar population of metallicity $Z=0.004$ and different values of $E(B-V)$ are superimposed. In each isochrone the contribution of the Galactic foreground reddening is taken into account. The sources in the black box have been used to study the spatial distribution of RC stars. The color scale is the same as in Figure 16.

reddening and are likely in front of 30 Dor, the majority of the stars suffer from a much higher reddening. It is interesting to note that the color of RC stars correlates with the distribution of the ionized gas on the eastern side of the Nebula, but not on the western side.

To the east (lower left corner of Figure 19) the reddest RC stars coincide with regions of high dust and gas concentrations. These are among the most extinguished RC stars with $E$ $(B-V)>0.8$ and coincides with the edges of hot super-bubbles \#1 and \#2 (see also Figure 1 for the location of the superbubbles). Inside the bubbles the interstellar medium (ISM) becomes more transparent $(E(B-V) \sim 0.3)$, suggesting a filamentary distribution for the gas with a low volume-filling factor. 


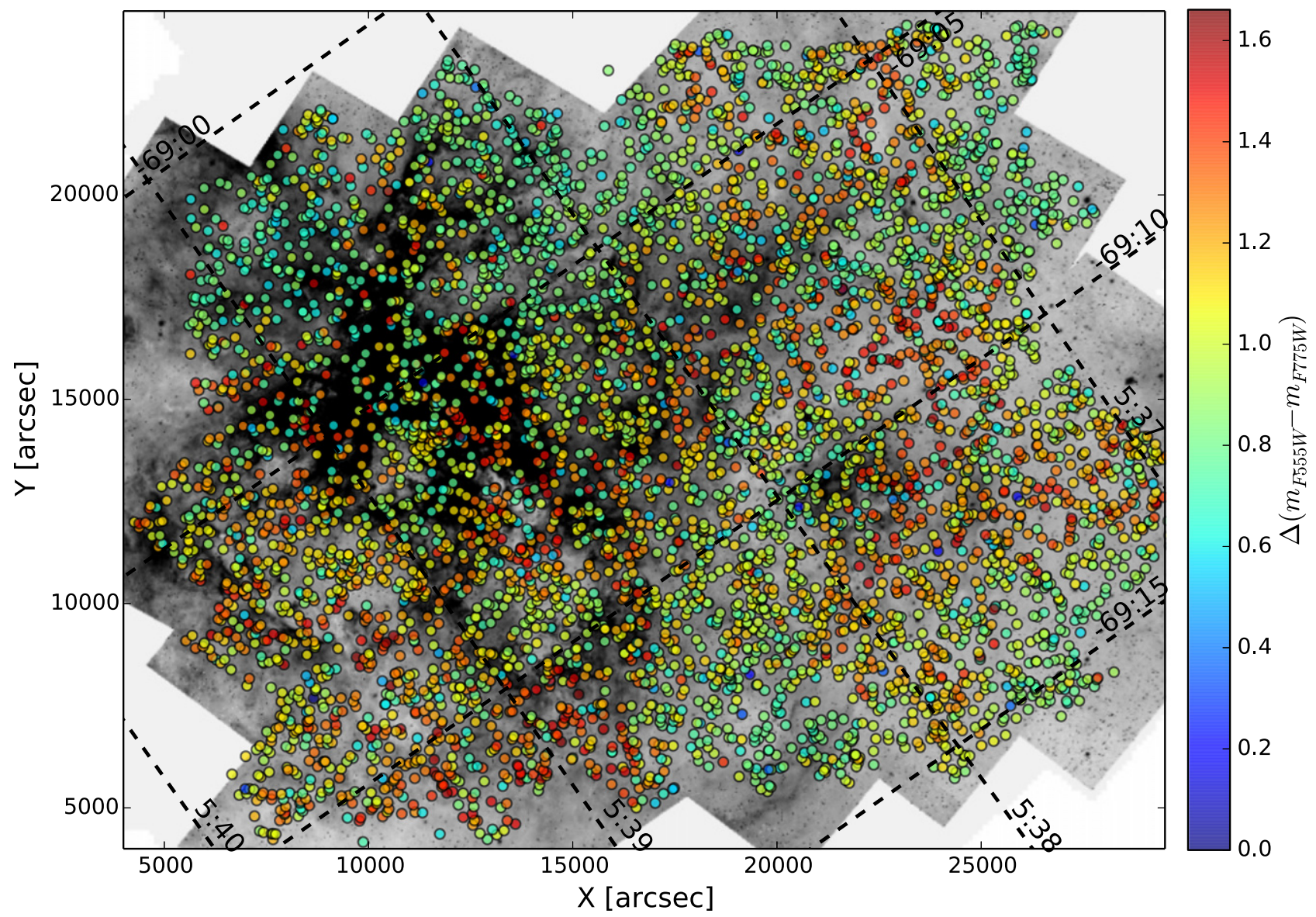

Figure 19. Spatial distribution of the RC stars selected from the $m_{\mathrm{F} 555 \mathrm{~W}}$ vs. $m_{\mathrm{F} 555 \mathrm{~W}}-m_{\mathrm{F} 775 \mathrm{~W}} \mathrm{CMD}$. Sources have been color-coded according to their of their color excess in the $m_{\mathrm{F} 555 \mathrm{~W}}-m_{\mathrm{F} 775 \mathrm{~W}}$ color, with the zero point of the distribution being the color of the RC affected only by the Galactic reddening.

On average, the region north to NGC $2070(x \leqslant 18,000$ and $y \geqslant 15,000)$, corresponding to hot super bubble \#5, Hodge 301 and hot super bubble \#4, is characterized by the lowest $(E$ $(B-V)<0.2)$ extinction. Another region where the ISM is relatively transparent is hot super-bubble \#3. Supernovae explosions and stellar winds are probably responsible for having removed the majority of the dust from these regions. Chandra X-rays images (Townsley et al. 2006), for example, show that hot super-bubble $\# 5$ is a chimney and that hot plasma is escaping toward the north.

The west and south portions of 30 Doradus do not show high concentrations of ionized gas (see, for example, Figure 3, panel (d)). Although on the majority of the region the ISM is relatively transparent $(E(B-V)<0.3)$, in some areas (i.e., to the west and the south of NGC 2060) the background stars are completely blocked.

Several of the dark clouds in Figure 19 have basically no blue RC stars. Since RC stars are too old to be associated with 30 Doradus, it is possible that at least these parts of the 30 Doradus system are above the majority of the LMC's stellar disk, suggesting a possible small offset of the Tarantula Nebula from the disk toward us. Similar offsets have been seen, for example, in the $\mathrm{H}$ II regions on NGC 55 (Ferguson et al. 1996). The possibility that 30 Doradus could be at the nearer side of the LMC was already proposed by Pellegrini et al. (2011) and Sabbi et al. (2013).
In De Marchi et al. (2016), we used the $2500 \mathrm{RC}$ stars present in the HTTP field to further investigate the extinction properties of the ISM. Following the same approach used by De Marchi et al. (2014) and De Marchi \& Panagia (2014), we discussed local variations in the extinction law, and their relation to the presence/absence of massive hot stars. In another paper (H. Arab et al. 2016, in preparation), we will use a Bayesian fit of the spectral energy distributions from UMS and RGB stars to infer properties of the dust, such as the size of the grains and their three-dimensional distribution.

\section{SPATIAL DISTRIBUTION AND AGES OF THE STARS IN 30 DORADUS}

UMS stars are short-lived objects that can be used to highlight sites of recent star formation. Figure 20 shows the spatial distribution of UMS candidates, selected in the magnitude range between $15<m_{\mathrm{F} 775 \mathrm{~W}}<19$ and with colors bluer than $m_{\mathrm{F} 555 \mathrm{~W}}-m_{\mathrm{F} 775 \mathrm{~W}}<0.6$. Similar to what we have done in Figure 19, UMS stars have been superimposed on the 30 Doradus mosaic obtained in the filter F555W. The size of the circles is proportional to the apparent magnitude of the stars, with the larger symbols corresponding to the brighter sources.

Although rotation, binarity, and age spread can contribute to the broadening of the UMS, for the majority of the source in the HTTP catalog the excess in the $m_{\mathrm{F} 555 \mathrm{~W}}-m_{\mathrm{F} 775 \mathrm{~W}}$ color is 


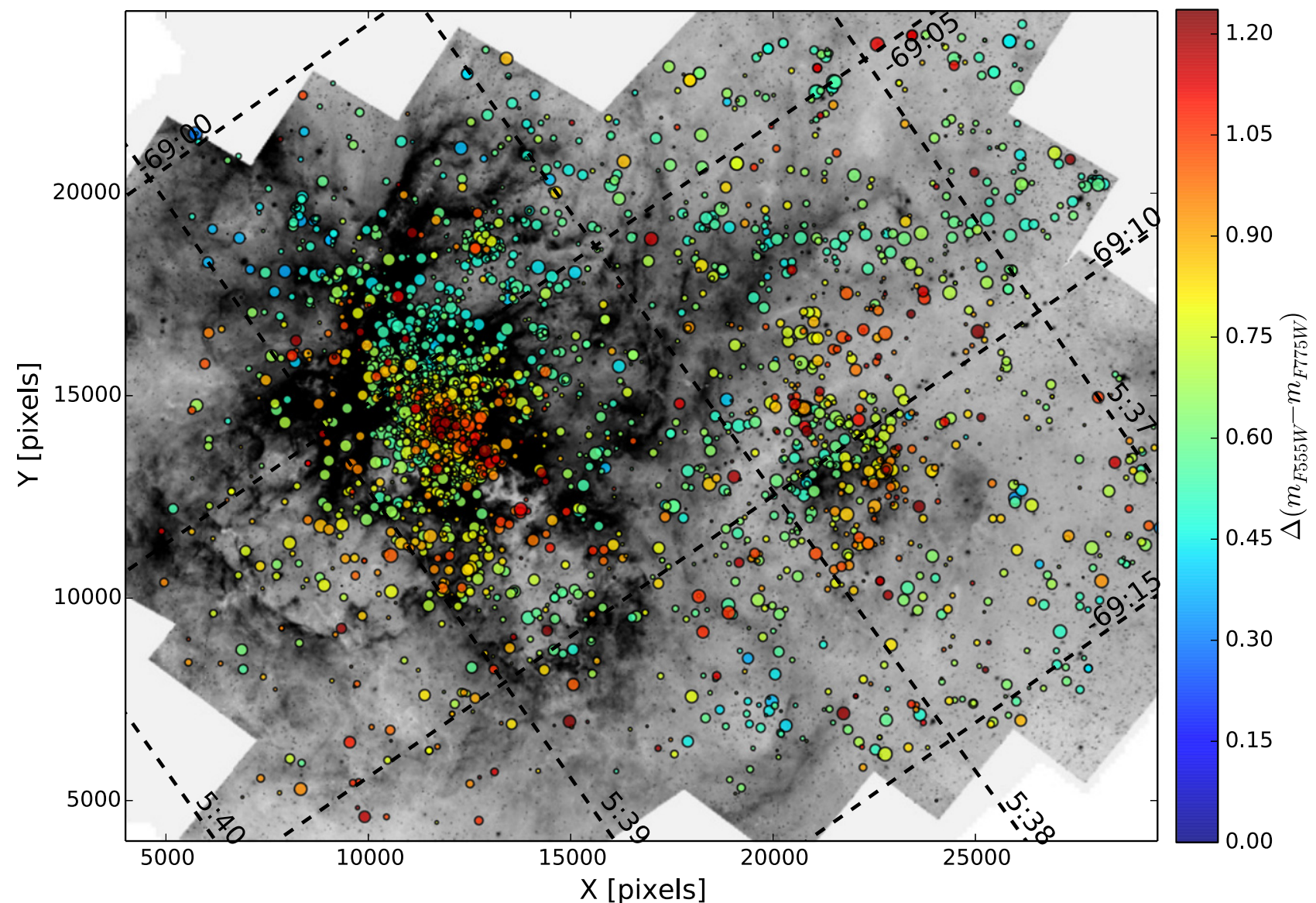

Figure 20. Spatial distribution of the UMS stars in the magnitude range between $15<m_{\mathrm{F} 775 \mathrm{~W}}<19$ and colors $-0.1<m_{\mathrm{F} 555 \mathrm{~W}}-m_{\mathrm{F} 775 \mathrm{~W}}<0.6$, overlaid on the image of 30 Doradus obtained in the ACS filter F555W. The size of the circles relates to the luminosity of the source in the F775W filter. Stars are color-coded according to their color excess relative to the color of UMS stars affected only by the galactic extinction.

likely proportional to the amount of extinction. To have a sense of how much the reddening is changing across the Tarantula Nebula, we color-coded the UMS stars from dark blue to red on the basis of their color excess with the respect of $m_{\mathrm{F} 555 \mathrm{~W}}$ $-m_{\mathrm{F} 775 \mathrm{~W}}$ color of UMS stars affected only by the galactic extinction. To do so, we had to rectify the UMS by deriving its fiducial line and then subtracting the color of the fiducial line at the corresponding F775W magnitude from the color of each selected UMS star.

As mentioned in the previous section, RC stars (Figure 19) trace the distribution of the old ( $>1-2$ Gyr) field of the LMC, and these stars are uniformly distributed over the entire region. The distribution of the UMS stars in Figure 20 instead is variable and complex, preserving at least partial memory of where the stars formed. The majority of the UMS stars are found in the three most prominent clusters and associations Hodge 301, NGC 2060, and the mini-starburst NGC 2070 (Leitherer 1998). In addition, several small associations of UMS stars can be found along the extended filaments of gas and dust between NGC 2070 and Hodge 301, around the edges of hot super-bubble $\# 5$, and inside the two hot super-bubbles \#1 and \#3. Finally, chains of massive blue stars can be found along the giant ( $\sim 140 \mathrm{pc}$ long) arc of dust and gas that divides the region in two parts and clearly separates NGC 2070 from NGC 2060.

Optical and NIR CMDs for the three main clusters are shown in Figure 21. For each cluster, we computed the ridge line of the UMS in the optical CMD and then fitted it with Padova isochrones to derive an estimate of the reddening. We then constrain the age of the cluster using the luminosity of the TOn. We then used the derived reddening and age to superimpose the isochrone on the NIR CMD. For all the clusters, we assumed a distance modulus of $(m-M)_{0}=18.5$, as proposed by Panagia et al. (1991) and Pietrzyński et al. (2013).

R136. R136 (R.A. ${ }_{J 2000}=05^{\mathrm{h}} 38^{\mathrm{m}} 42^{\mathrm{s}} \cdot 3$ decl. ${ }_{J 2000}=-69^{\circ} 06^{\prime}$ 03 ". 3 ) is the youngest cluster in the 30 Doradus region. It is at the center of the mini-starburst NGC 2070 and contains the most massive stars known so far (Crowther et al. 2010). R136's 10 brightest stars are responsible for almost $30 \%$ of the entire ionizing flux in 30 Doradus (Doran et al. 2013). The structure of NGC 2070 is complex and includes multiple clusters and associations of different ages and sizes (Walborn \& Bladesm 1997). Selman et al. (1999) found that NGC 2070 likely formed stars over a relatively prolonged interval of time, with star formation progressing from the outside in.

Recently, Sabbi et al. (2012) noted that NGC 2070 can be separated into two components: a slightly older elongated and more diffuse system extending toward the northeast (called the NE clump), and the very compact and younger R136. The two top panels of Figure 21 show the optical and NIR CMDs for stars within $5 \mathrm{pc}$ from R136 (thus excluding the contribution of the NE clump).

From isochrone fitting we found that R136 is affected by a relatively high amount of reddening, with $E(B-V)=0.35$ and 

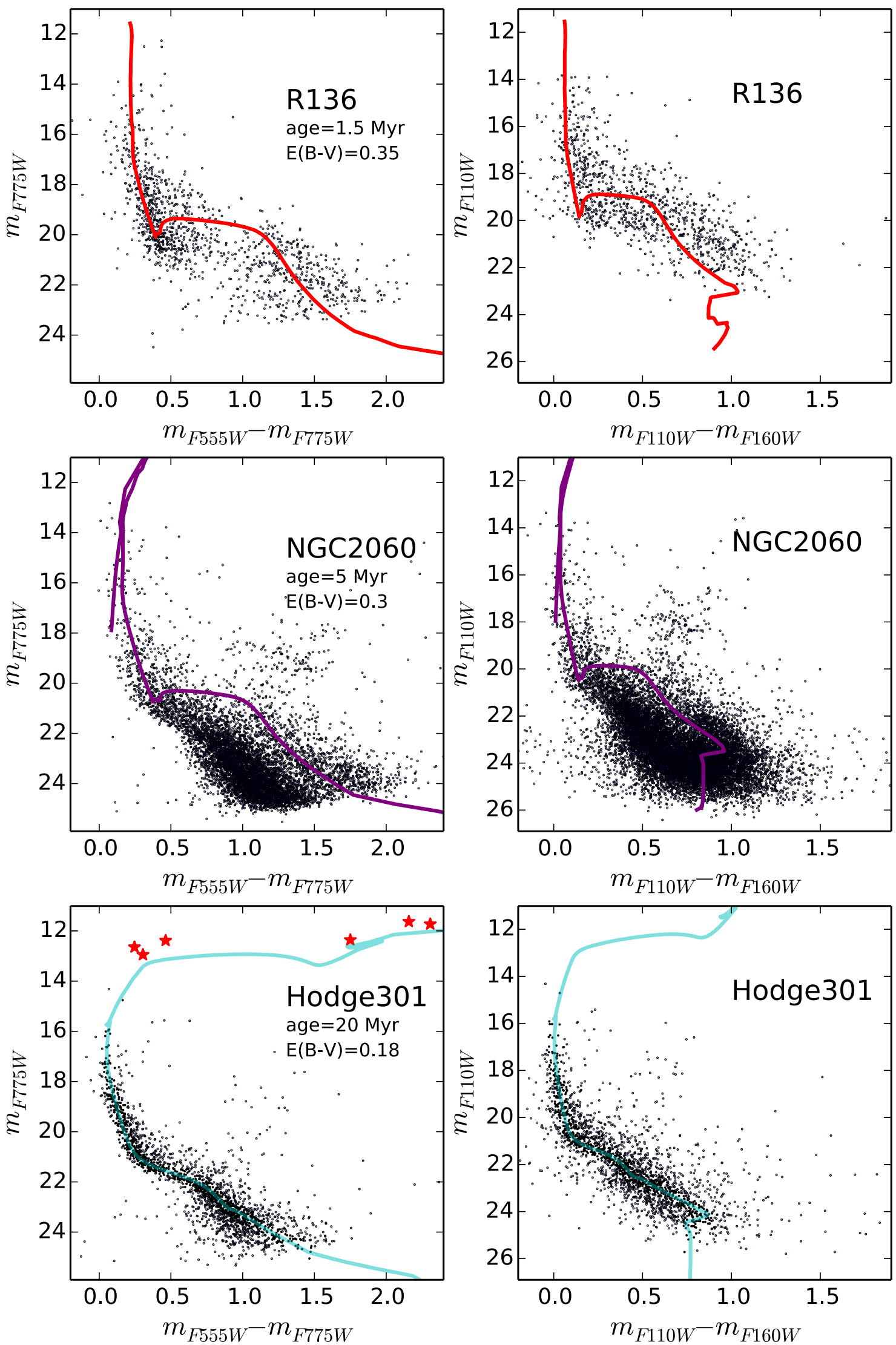

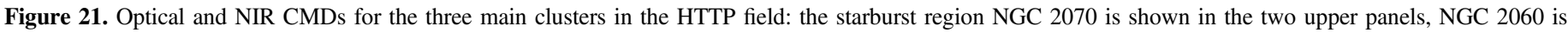

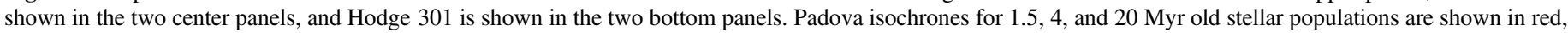
purple, and cyan, respectively. Red stellar symbols in the optical CMD of Hodge 301 highlight the position of the blue and red supergiants. 
formed most of its stars between 1 and $4 \mathrm{Myr}$ ago, although some star formation could be still ongoing, in agreement with the findings of Rubio et al. (1992), Walborn \& Bladesm (1997), Massey \& Hunter (1998), and Selman et al. (1999). In addition, a multitude of embedded objects at the top of extended pillars, as well as dark Bok globules, surrounding R136 could be the result of a very recent episode of star formation triggered by the interaction of the stellar winds from R136 and the supernova remnants associated with Hodge 301 as suggested by Walborn \& Bladesm (1997). In Cignoni et al. (2015), we compared HTTP data with synthetic CMDs to reconstruct the SFH of NGC 2070 over the last 20 Myr. In doing so, we found that the peak of star formation occurred in the last $3 \mathrm{Myr}$, even if within the $20 \mathrm{pc}$ from the center of R136 the star formation rate was significantly above the average value of the LMC already 7 Myr ago.

To reproduce the colors of the stars in the UMS in Figure 21, we had to assume a higher reddening value than what we used in the UV (Figure 14). This difference is likely an artifact due to the gap in the F275W mosaic (Figures 3 and 4, panel (a)). Because of the gap, in fact, the NUV CMD covers only the northern part of NGC 2070, characterized by a lower extinction.

$N G C$ 2060. NGC 2060 (R.A. $J 2000=5^{\mathrm{h}}: 37^{\mathrm{m}}: 46^{\mathrm{s}} \cdot 9$; decl. $\cdot 2000=$ $\left.-69^{\circ}: 10^{\prime}: 18^{\prime \prime}\right)$ is an extended $(r \simeq 21 \mathrm{pc})$ star-forming region that hosts the young ( 5000 year), fast-rotating X-ray pulsars PSR J0537-6910 (Cusumano et al. 1998; Marshall et al. 1998) and many Wolf-Reyet (WR) stars (Breysacher 1981). Part of the region is obscured by a bird-like dark cloud of dust, which makes it extremely difficult to characterize the spatial distribution and stellar density of the system.

The reddening varies considerably across the system, with the ISM becoming more transparent in the peripheral areas, particularly toward the northeast. To estimate the age of NGC 2060, we selected the stars within $\sim 5 \mathrm{pc}$ from R.A. $=5^{\mathrm{h}}: 37^{\mathrm{m}}: 51^{\mathrm{s}} .6$; decl. $=-69^{\circ}: 10^{\prime}: 22^{\prime \prime} \cdot 3$ and around the compact cluster that hosts the WR stars Br73 (Figure 21, two center panels). In this region, the average $E(B-V)=0.3$ and the cluster is likely $5 \pm 1$ Myr old, confirming the young ages proposed by Schild \& Testor (1992) and Walborn et al. (1995), who spectroscopically identified several early O-type stars across the region.

Hodge 301. Hodge 301 (R.A. $J 2000=5^{\mathrm{h}}: 38^{\mathrm{m}}: 17^{\mathrm{s}}$; decl. $J 2000=$ $\left.-69^{\circ}: 04^{\prime}: 00^{\prime \prime}\right)$ is the older cluster in the Tarantula Nebula. Compared to the previous systems, the reddening across the region is more uniform and relatively low $(E(B-V)=0.18)$, probably because stellar winds first and supernova explosions later (based on the present day stellar mass function, Grebel \& Chu 2000 estimated that the cluster may have survived up to 40 supernova explosions) have cleaned the region from most of the dust.

The Hodge 301 optical and NIR CMDs are shown in the two lower panels of Figure 21. Colors and luminosities of the bright red supergiants $\left(1.6<m_{\mathrm{F} 555 \mathrm{~W}}-m_{\mathrm{F} 775 \mathrm{~W}}<2.2\right.$, $\left.13.8<m_{\mathrm{F} 775 \mathrm{~W}}<14.3\right)$ and the blue supergiants $(0.15<$ $\left.m_{\mathrm{F} 555 \mathrm{~W}}-m_{\mathrm{F} 775 \mathrm{~W}}<0.3,12.9<m_{\mathrm{F} 775 \mathrm{~W}}<13.3\right)$ stars can be reproduced with A Padova isochrones for metallicity $Z=0.008$, and ages between 16 and $20 \mathrm{Myr}$, in excellent agreement with the age derived by Evans et al. (2015) from the spectroscopic analysis of $15 \mathrm{~B}$-type stars. It is interesting to note that this age estimate is in good agreement with the values obtained by Grebel \& Chu (2000) using the isochrones from
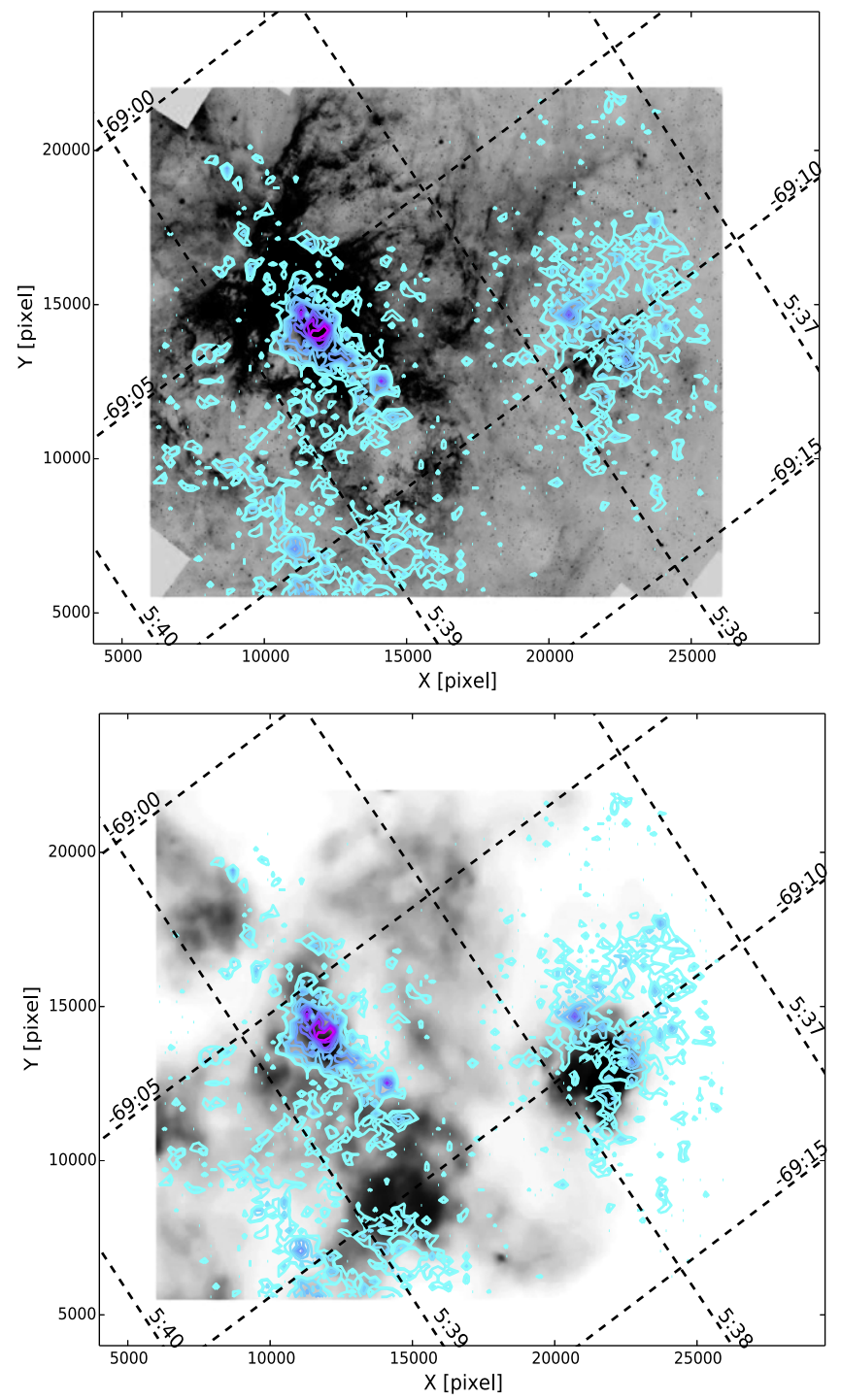

Figure 22. Spatial density distribution of PMS candidates. From purple to cyan, contours show 99\%, 97.5\%, 96.5\%, 95\%, 93\%, 90\% 85\%, 80\%, 65\%, and $50 \%$ of the PMS candidates. Contours are overlaid on a portion of the HTTP mosaic in the F555W filter (upper panel) and a portion of the Chandra/ ACIS image of 30 Doradus (lower panel).

the Geneva group (Schaerer et al. 1993), but younger than the age derived by the same team using an older set of Padova isochrones (Bertelli et al. 1994).

It is also worth noting that the new set of Padova isochrones fails to reproduce the characteristics of the low-mass stars, whose colors and magnitudes in both the optical and IR CMDs are more consistent with those of a $\sim 25 \mathrm{Myr}$ old stellar population. This discrepancy is not surprising because the evolutionary time for the PMS phase are still very uncertain.

Furthermore, recent studies in both the Milky Way (Sana et al. 2012) and 30 Dor (Sana et al. 2013; Dunstall et al. 2015) show that most of the massive $\mathrm{O}$ and $\mathrm{B}$ stars are close binaries. In Hodge 301, however, the fraction of OB-type binaries is significantly lower than in the rest of the Tarantula Nebula (Dunstall et al. 2015), possibly as a result of the binary dynamical evolution (de Mink et al. 2014). If this is the case, the direct comparison of UMS stars in Hodge 301 with single star evolutionary models would be hazardous. Indeed, mergers and mass transfer can artificially rejuvenate the most massive 
stars, creating an apparent discrepancy between these objects and the sources at lower masses already 5 Myr after the cluster formation (Schneider et al. 2014).

\subsection{PMS Stars and Evidence for Triggered Star Formation}

As mentioned in Section 5.2, most of the faint and red objects in both the optical and in the NIR CMDs (Figures 16 and 17) are likely PMS stars. Because of their young age, PMS stars have not had time to migrate far away from their birth place, and therefore their spatial distribution can be used to trace how star formation occurred across a region. For this purpose, in the optical CMD we selected all the sources to the right of the Padova $Z=0.0085 \mathrm{Myr}$ old isochrone (assuming the distance modulus 18.5 and $E(B-V)=0.3$ ), in the magnitude range $21.5<m_{m_{\mathrm{F} 775 \mathrm{~W}}}<23.0$ and bluer than $m_{\mathrm{F} 555 \mathrm{~W}}-m_{\mathrm{F} 775 \mathrm{~W}}<2.0$.

PMS candidates can be found almost everywhere in the region (see Figure 4 in Cignoni et al. 2015), although clear overdensities can be noted, for example, in correspondence of the two young clusters NGC 2060 and NGC 2070. Because highly extinguished MS and SGB stars can contaminate the distribution of PMS candidates, in Figure 22 we show the density contours only for regions whose number of PMS candidates is above the 50 percentile. This selection allows us also to highlight the clustering properties of these sources (Lada \& Lada 2003). To help with the interpretation in the upper panel, we superimposed the density contours on the mosaic acquired in the F555W filter.

The figure shows an excellent correlation between the distribution of the PMS candidates and the ionized filaments of ionized gas and warm dust that envelope and confine several extended hot $\left(10^{6}-10^{7} \mathrm{~K}\right)$ bubbles of plasmas. The lower panel of Figure 22 shows that PMS candidates also correlate well with soft X-rays detected by Chandra ACIS at 700-1120 eV (Townsley et al. 2006). In particular we find the following.

1. To the north, chains of small clusters clearly depart from NGC 2070 and envelop the giant hot super bubble \#5. The densest point in this system is only partially resolved into stars by HST and is still heavily embedded in its dusty cocoon. This system, nicknamed the "Skull Nebula," is also one of the brightest sources in mid-IR (Walborn. et al. 2013).

2. The boundary between hot super-bubbles \#1 and \#2, as well as the east side of hot super-bubble \#2 are marked by several clumps of PMS candidates.

3. PMS stars surround NGC 2060. The two richer systems coincide with diffuse soft X-rays to the north and the east of the SNR, the larger being associated with the compact bright cluster hosting Br73.

4. The large arc of gas and dust that divides NGC 2060 and NGC 2070 in the X-ray is emitting at 700-1120 eV and is the birthsite of several compact associations. The X-Ray peak of hot super-bubble \#4, on the contrary, occurs at $350-700 \mathrm{eV}$ and the region appears devoid of PMS stars.

The clumps of PMS candidates identified with HTTP also correlate with the population of embedded young stellar objects (YSOs) identified by Seale et al. (2014) combining Herschel and Spitzer data. The strong correlation between cool gas, warm dust, and PMS candidates, as well as the anti-correlation between hot gas and very recent star formation, once again shows how, by carving cavities in the ISM, strong stellar winds from massive stars, WR stars, and supernovae explosions, shut down star formation (disruptive feedback) in some regions, and ignite new generations of stars several parsecs away (constructive feedback).

\section{CONCLUSIONS}

HTTP (HST GO-12939, PI: E. Sabbi) is a multi-wavelength study of 30 Doradus, the closest extragalactic giant $\mathrm{H}$ II region and an excellent example of large-scale star formation in an environment that, in many ways (such as metallicity, dust content and SF rate), resembles the extreme conditions of the early universe. HTTP covers the 30 Dor region in the NUV (F275W, F336W), optical (F555W, F658N, F775W), and NIR (F110W, F160W) wavelengths using simultaneously both the imagers (WFC3 and ACS) currently operating on HST.

In this paper, we have presented the observing strategy, the data analysis, and the photometric catalog for more than 600,000 stars. The stellar photometry was measured by PSF fitting all the filters simultaneously. The astrometric rms are less then 0.4 mas. The completeness of the catalog varies across the field depending both on crowding and background values.

Since HTTP is a Treasury project, all the data collected have been immediately available to the astronomical community. To enhance the scientific return of HTTP, we are now releasing the astro-photometric catalog, results from the artificial star tests, and the mosaicked images for all the HTTP's filters for download from the ApJS, ADS, and the MAST websites.

Our study probes the stellar content of the Tarantula Nebula down to $\sim 0.5 M_{\odot}$ and provides a snapshot of the history of star formation of the entire region, confirming that 30 Dor is a complex region that has built up its stellar content over several million years. The oldest stellar population observed at all wavelengths belongs to the field of the LMC. This component is uniformly distributed over the entire region. The presence of RGB and RC stars confirms the finding of Zaritsky et al. (2004) that the field has been forming stars for several billion years, and that it has been relatively active even in the recent past.

The spread in color of RC stars indicates that extinction is variable, with some areas totally blocking the background and others being transparent. This suggests the nebula is a partially filled structure offset toward the near side of the local LMC stellar disk.

The younger stellar population is organized in several clusters and associations. The larger systems in order of increasing age are NGC 2070, NGC 2060, and Hodge 301. In using Padova isochrones to obtain a first estimate of the clusters ages, we find that NGC 2070 has likely formed the majority of its stars in the last $3 \mathrm{Myr}$ and that the majority of the stars in NGC 2060 are likely as young as $~ 5 \mathrm{Myr}$ in agreement with previous photometric and spectroscopic studies.

The comparison of the UV CMDs with theoretical models shows that at these wavelengths the models of atmosphere fail to reproduce the colors of the PMS candidates. The models and observations are in very good agreement in the optical and in the NIR. It is similarly possible that theoretical models fail to properly reproduce the characteristics of low-mass stars at older ages. For example, in the case of Hodge 301 Padova isochrones were not able to reproduce at the same time the properties of high- and low-mass stars in the optical, implying a difference in age between the brightest stars and the low-mass stars as large as $8-10 \mathrm{Myr}$. On the other hand if the models are correct, then the most massive stars could be the by-product of 
mergers and mass transfer in binary systems and appear younger than their real age, like in the case of the blue stragglers stars (Sandage 1953) found older clusters.

While several studies have focused on Tarantula's massive stars, HTTP has provided for the first time a rich and statistically significant census of the low-mass PMS stars. Although the complicated kinematics (Chu \& Kennicutt 1994) and the highly variable reddening make it difficult to infer the tridimensional structure of 30 Dor, the high spatial resolution and sensitivity of HST have allowed us to trace how star formation has been developing in the region.

The distribution of UMS stars and PMS candidates in clumps and filaments mimics the predictions of recent hydrodynamical simulation (i.e., Bate 2012; Krumholz et al. 2012; Schneider et al. 2012), supporting the hypothesis that stars may preferentially form in those filaments of gas that built up more mass during the turbulent formation of the cloud. In addition, the fact that PMS candidates surround many of the super-bubbles carved by supernova explosions suggests that fast supernova explosions had a major role in triggering the most recent episodes of star formation and that stellar feedback is still shaping the region. The overlap between PMS candidates and embedded YSOs indicates that, once initiated, the process of star formation continues for at least a few million years, even in the presence of fast stellar winds and high UV radiation, and that the SF episode in 30 Dor is not complete and is continuing to evolve.

We thank the anonymous referee for the thorough review and highly appreciate the comments and suggestions which significantly contributed to improving the quality of this paper. We thank Karen Levay and the MAST HLSP Team at STScI for their invaluable help in releasing to the astronomical community all the high level science products associated with HTTP. Based on observations with the NASA/ESA Hubble Space Telescope, obtained at the Space Telescope Science Institute, which is operated by AURA Inc., under NASA contract NAS 5-26555. These observations were associated with Programs 12499 and 12939. Support for both Programs 12499 and 12939 was provided by NASA through grants from the Space Telescope Science Institute. D.A.G. kindly acknowledges financial support by the German Research Foundation (DFG) through grant GO 1659/3-2. S.d.M. acknowledges support by the European Commission, grant H2020-MSCAIF-2014, project ID 661502. M.T. was partially funded by the Italian MIUR through the grant PRIN-MIUR 2010LY5N2T. E. K.G. acknowledges support by Sonderforschungsbereich SFB 881 "The Milky Way System" of the German Research Foundation (DFG), particularly subproject B5.

Facilities: HST(WFC3), HST(ACS).

\section{REFERENCES}

Andersen, M., Zinnecker, H., Moneti, A., et al. 2009, ApJ, 707, 1347 Anderson, J., \& King, J. R. 2006, STSCI Institute Science Report ACS 200601 (Baltimore, MD: STScI)

Anderson, J., Sarajedini, A., Bedin, L. R., et al. 2008, AJ, 135, 2055

Baggett, S., \& Anderson, J. 2012, STSCI Institute Science Report WFC3 2012 12 (Baltimore, MD: STScI)

Bate, M. R. 2012, MNRAS, 419, 3115

Bernard, J.-P., Reach, W. T., Paradis, D., et al. 2008, AJ, 136, 919

Bertelli, G., Bressan, A., Chiosi, C., Fagotto, F., \& Nasi, E. 1994, A\&AS, 106,275

Bestenlehner, J. M., Vink, J. S., Gräfener, G., et al. 2011, A\&A, 530, L14
Bosch, G., Selman, F., Melnick, J., \& Terlevich, R. 2001, A\&A, 380, 137 Bourque, M., \& Kozurina-Platais, V. 2013, STSCI Institute Science Report WFC3 2013-03 (Baltimore, MD: STScI)

Brandner, W., Grebel, E. K., Barbá, R. H., Walborn, N. R., \& Moneti, A. 2001, AJ, 122, 858

Bressan, A., Marigo, P., Girardi, L., et al. 2012, MNRAS, 427, 127

Breysacher, J. 1981, A\&AS, 43, 203

Chen, Y., Girardi, L., Bressan, A., et al. 2014, MNRAS, 444, 2525

Chu, Y.-H., \& Kennicutt, R. C., Jr. 1994, AJ, 108, 1696

Cignoni, M., Sabbi, E., van der Marel, R. P., et al. 2015, ApJ, 811, 76 Cioni, M.-R., Clementini, G., Girardi, L., et al. 2011, A\&A, 527A, 116 Crowther, P. A., Schnurr, O., Hirschi, R., et al. 2010, MNRAS, 408, 731 Cusumano, G., Maccarone, M. C., Mineo, T., et al. 1998, A\&A, 333, L55 de la Caille, N. L. 1761, RSPT, 52, 21

De Marchi, G., \& Panagia, N. 2014, MNRAS, 445, 1

De Marchi, G., Panagia, N., \& Girardi, L. 2014, MNRAS, 438, 1

De Marchi, G., Panagia, N., Sabbi, E., et al. 2016, MNRAS, 455, 4373

De Marchi, G., Paresce, F., Panagia, N., et al. 2011, ApJ, 739, 27

de Mink, S. E., Sana, H., Langer, N., Izzard, R. G., \& Schneider, F. R. N. 2014, ApJ, 782, 7

Doran, E. I., Crowther, P. A., de Koter, A., et al. 2013, A\&A, 558, A134

Dufour, R. J., Shields, G. A., \& Talbot, R. J., Jr. 1982, ApJ, 252, 461

Dunstall, P. R., Dufton, P. L., Sana., H., et al. 2015, A\&A, 580, A93

Evans, C. J., Kennedy, M. B., Dufton, P. L., et al. 2015, A\&A, 574, 13

Evans, C. J., Taylor, W., Hénault-Brunet, V., et al. 2011, A\&A, 530A, 108

Evans, C. J., Walborn, N. R., Crowther, P. A., et al. 2010, ApJL, 715, L74

Ferguson, A. M. N., Wyse, R. F. G., \& Gallagher, J. S. 1996, AJ, 112, 256

Gilliland, R. 2004, STSCI Institute Science Report ACS 2004-01 (Baltimore, MD: STScI)

Gilliland, R. L., Rajan, A., \& Deustua, S. 2010, STSCI Institute Science Report WFC3 2010-10 (Baltimore, MD: STScI)

Girardi, L., \& Salaris, M. 2001, MNRAS, 323, 109

Gouliermis, D. 2012, SSRv, 169, 1

Grebel, E. K., \& Chu, Y.-H. 2000, AJ, 111, 787

Gullbring, E., Hartmann, L., Briceno, C., \& Calvet, N. 1998, ApJ, 492, 323

Haschke, R., Grebel, E. K., \& Duffau, S. 2011, AJ, 141, 158

Heckman, T. M., Kauffmann, G., Brinchmann, J., et al. 2004, ApJ, 613, 109

Herschel, J. F. W. 1847, Results of Astronomical Observations Made during the Years 1834, 5, 6, 7, 8, at the Cape of Good Hope; Being the Completion of a Telescopic Survey of the Whole Surface of the Visible Heavens, Commenced in 1825 (London: Smith, Elder, Co.)

Hog, E., Fabricius, C., Makarov, V. V., et al. 2000, A\&A, 355, L27

Hunt, L. K., \& Hirashita, H. 2009, A\&A, 507, 1327

Hunter, D., O’Neil, E. J., Lynds, R., et al. 1996, ApJL, 459, L27

Hunter, D., Shaya, E. J., Holtzman, J. A., et al. 1995, ApJ, 448, 179

Kennicutt, R. C., Edgar, B. K., \& Hodge, P. W. 1989, ApJ, 337, 761

Kennicutt, R. C., \& Hodge, P. W. 1986, ApJ, 306, 130

Krumholz, M. R., Klein, R. I., \& McKee, C. F. 2012, ApJ, 754, 71

Kuhi, L. V. 1974, A\&AS, 15, 47

Lada, C. J., \& Lada, E. A. 2003, ARA\&A, 41, 57

Leitherer, C. 1998, in Stellar Astrophysics for the Local Group: VIII Canary Islands Winter School of Astrophysics, ed. A. Aparicio, A. Herrero \& F. Sanchez (Cambridge: Cambridge Univ. Press), 527

Long, K. S., Baggett, S. M., \& MacKenty, J. W. 2013a, STSCI Institute Science Report WFC3 2013-06 (Baltimore, MD: STScI)

Long, K. S., Baggett, S. M., \& MacKenty, J. W. 2013b, STSCI Institute Science Report WFC3 2013-07 (Baltimore, MD: STScI)

Marshall, F. E., Gotthelf, E. V., Zhang, W., Middleditch, J., \& Wang, Q. D 1998, ApJL, 499, L179

Massey, P., \& Hunter, D. A. 1998, ApJ, 493, 180

Meixner, M., Galliano, F., Hony, S., et al. 2010, A\&A, 518, L71

Meixner, M., Gordon, K. D., Indebetouw, R., et al. 2006, AJ, 132, 2268

Meurer, G. R., Heckman, T. M., Lehnert, M. D., Leitherer, C., \& Lowenthal, J. 1997, AJ, 114, 54

Mignani, R. P., Pulone, L., Iannicola, G., et al. 2015, A\&A, 583, A105 Nikolaev, S., Drake, A. J., Keller, S. C., et al. 2004, ApJ, 601, 260

Oey, M. S., Parker, J. S., Mikles, V. J., \& Zhang, X. 2003, AJ, 126, 2317

Panagia, N., Gilmozzi, R., Macchetto, F., Adorf, H.-M., \& Kirshner, R. P. 1991, ApJL, 380, L23

Pellegrini, E. W., Baldwin, J. A., \& Ferland, G. J. 2011, ApJ, 738, 34

Pietrzyński, G., Graczyk, D., Gieren, W., et al. 2013, Natur, 495, 76

Platais, I., van der Marel, R. P., Lennon, D. J., et al. 2015, AJ, 150, 89

Robberto, M., Song, J., Mora Carrillo, G., et al. 2004, ApJ, 606, 952

Rubio, M., Barbá, R. H., \& Walborn, N. R. 1999, A\&A, 347, 532

Rubio, M., Roth, M., \& Garcia, J. 1992, A\&A, 261, L29

Sabbi, E., Anderson, J., Lennon, D. J., et al. 2013, AJ, 146, 53 
Sabbi, E., Lennon, D. J., Gieles, M., et al. 2012, ApJL, 754, L37

Sana, H., de Koter, A., de Mink, S. E., et al. 2013, A\&A, 550, 107

Sana, H., de Mink, S. E., de Koter, A., et al. 2012, Sci, 337, 444

Sandage, A. R. 1953, AJ, 58, 61

Schaerer, D., Meynet, G., Maeder, A., \& Schaller, G. 1993, A\&AS, 98, 523

Schild, H., \& Testor, G. 1992, A\&AS, 92, 729

Schneider, F. R. N., Izzard, R. G., de Mink, S. E., et al. 2014, ApJ, 780, 117

Schneider, N., Csengeri, T., Hennemann, M., et al. 2012, A\&A, 540, L1

Seale, J. P., Meixner, M., Sewilo, M., et al. 2014, AJ, 148, 124

Selman, F. J., \& Melnick, J. 2013, A\&A, 552, A94

Selman, F. J., Melnick, J., Bosch, G., \& Terlevich, R. 1999, A\&A, 347, 532

Shapley, A. E., Steidel, C. C., Pettini, M., \& Adelberg, K. L. 2003, ApJ, 588,65

Skrutskie, M. F., Cutri, R. M., Stiening, R., et al. 2006, AJ, 131, 1163
Stanek, K. Z., Zaritsky, D., \& Harris, J. 1998, ApJL, 500, L141

Tang, J., Bressan, A., Rosenfield, P., et al. 2014, MNRAS, 445, 4287

Townsley, L. K., Broos, P. S., Feigelson, E. D., Garmire, G. P., \& Getman, K. V. 2006, AJ, 131, 2140

Udalski, A., Soszyns̀ki, I., Szymanski, M., et al. 1999a, AcA, 49, 223

Udalski, A., Soszyns̀ki, I., Szymanski, M., et al. 1999b, AcA, 49, 437

Walborn, N. R., Barbá, R. H., Brandner, W., et al. 1999, AJ, 117, 225

Walborn., N. R., Barbà, R. H., \& Sewilo, M. M. 2013, AJ, 145, 98

Walborn, N. R., \& Bladesm, J. C. 1997, ApJS, 112, 457

Walborn, N. R., MacKenty, J. W., Saha, A., White, R. L., \& Parkerm, J. W. 1995, ApJL, 439, L47

Walborn, N. R., Maíz Apellániz, J., \& Barbá, R. H. 2002, AJ, 124, 1601

Wang, Q., \& Helfand, D. J. 1991, ApJ, 370, 541

Zaritsky, D., Harris, J., Tomphson, I. B., \& Grebel, E. K. 2004, AJ, 128, 1606 